\title{
Méthode de prédétermination des caractéristiques électromagnétiques de machines à bobinage global à commutation de flux. Application à un actionneur linéaire
}

\author{
L. Prevond (*), A. Ben Ahmed, B. Multon et J. Lucidarme \\ Laboratorre d'Électrıcité, Signaux et Robotique (LES1R) $\left({ }^{* *}\right)$, ENS-Cachan, \\ École Normale Supérieure de Cachan, 61 avenue du président Wilson, \\ 94235 Cachan Cedex, France
}

(Reçu le 3 octobre 1996, révısé le 3 janvier et le 4 mars 1997, accepté le 11 mars 1997)

PACS.02.60 - $\mathrm{x}$ - Numerical approxımation and analysis

\begin{abstract}
Résumé. - Cet article présente une méthode de prédétermmation des caractérıstiques de machines multicellulaires (bobinage global) à commutation de flux (aimants permanents) en tenant compte de la non linéarité des matériaux ferromagnétıques. Cette méthode allie le calcul numérique par éléments finıs et le calcul analytique. Cette étude nous a permis, d'une part, d'effectuer une analyse paramétrique intrınsèque et, d'autre part, de définir les paramètres optımaux de la cellule à commutation de flux. La notion de désaimantation est introduite en calculant le champ démagnétisant dans l'aimant. Les calculs effectués, dans le cas d'un actionneur linéarre à commutation de flux, montrent une bonne corrélation avec les essars directs
\end{abstract}

\begin{abstract}
This paper presents a predetermmation method of Permanent Magnet (P.M.) multicellular flux switchng machines. The non-linear characterıstic of the ferromagnetic material is integrated in this method which combines analytical and numerical calculus. This study permits to make a fast parametrical analysıs to define optimal parameters of a flux switching cell such as magnetic shear stress and flux concentration factor as function of magnetomotive force. The demagnetisation limit is introduced by a finite element calculus applied. The comparison of the theoritıcal results and experimental one gives a good correlation.
\end{abstract}

\section{Introduction}

Avec le développement d'aimants performants ou/et économiques et de l'électronique de puissance, de nombreuses structures non conventionnelles d'actionneurs électromécaniques se sont développées $[1,3]$. Des machines à réluctance variable et à aimants sont connues depuis 150 ans mais elles n'ont pu trouver des débouchés industriels que récemment, souvent dans des applications spécifiques et assez marginales. Les structures associant effet de réluctance variable et excitation par aimants permanents, communément qualifiées d'hybrides, permettent une très grande variété de structures [2] et il est encore possible d'en trouver de nouvelles $[7,8]$.

( ${ }^{*}$ ) Auteur auquel doit être adressée la correspondance (e-mail : prevond@lesir.ens-cachan.fr)

$\left({ }^{* *}\right)$ URA CNRS D1375

(C) Les Éditions de Physique 1997 
Une caractéristique importante qui est ici mise à profit est la possibilité d'une meilleure intégration de la structure électromagnétique à la fonction globale dans laquelle elle se trouve.

Dans la grande famille des machines hybrides, nous nous intéressons aux structures dites à commutation de flux $[4,7,8]$ dans lesquelles 1 est possible de solidariser inducteur (à aimants) et induit. Cette disposition permet notamment, dans le cas des machines tournantes d'avoir un rotor passif (sans aimants ni conducteur) et, dans le cas des actionneurs linéaires, de se satisfaire d'un rail également passif. C'est cette dernière qualité qui nous a intéressé pour la conception de l'actionneur linéaure décrit dans la suite de cet article. En effet, l'économie procurée, pour de grandes longueurs, par un simple rail en acier est tout à fait significative. En outre, la disposition des aimants dans le circuit magnétique d'induit rend possible une assez grande "concentration de flux" grâce à la place disponible. On peut ainsi utiliser des aimants en ferrite à faible coût sans trop grande dégradation des performances.

En contre partie, ces structures électromagnétiques sont généralement tridimensionnelles et très saturées $[5,8]$ ce qui complique notablement leur étude et leur optimisation [6]. Ces saturations (locales et globales) entraînent des caractéristiques magnétiques fortement nonlinéaires rendant difficiles et excessivement lourds les calculs et l'optimisation de ces structures par les méthodes classiques [10,11].

Nous proposons une modélisation de ces machines, alliant le calcul analytique et numérique par éléments finis et permettant la prédétermination rapide de leurs performances ainsi que leur optimisation en régime non-linéaire en tenant compte de manière simplifiée de certaines contraintes.

\section{Définitions et formalisme}

2.1. DÉfinition des machines À Commutation de flux. - De telles structures possèdent un aspect multicellulaire [9] permettant de polarıser plusieurs éléments actifs à partir d'un seul enroulement appelé bobinage global. Ceci permet notamment une augmentation du couple volumique grâce à l'accroissement du nombre de pôles et de la fréquence. Cela permet aussi la miniaturisation de certaines structures en facilitant la réalisation des bobinages solénoïdaux.

Le principe de la commutation de flux peut être présenté à partir d'un élément simple, appelé cellule élémentaire que nous représenterons, pour une compréhension plus aisée, dans le cas d'un actionneur linéaire. La cellule élémentaire est composée (Fig. 1a), de deux pièces ferromagnétiques (ou plots) (I), séparées par un ou plusieurs aimants (A), le tout étant enlacé par un bobinage (B). Le court-circuit magnétique des faces I1 et I2' est réalisé par une pièce ferromagnétique ou rail $(R)$ qui se positionne successivement entre les faces (I1, I2') et (I1', I2), comme indiqué sur les figures $1 \mathrm{~b}$ et 1c. Le bobinage est alors traversé par un flux alternatif lors du déplacement relatif de la pièce passive " $R$ " par rapport à la cellule élémentaire.

La figure 2a représente la coupe d'un prototype d'actionneur linéaire réalisé au LESiR sur lequel reposera la validation de la modélisation décrite dans cet article. La photographie de la figure $2 \mathrm{c}$ montre le rail ferromagnétique réalisant le court-circuit magnétique et entourant la partie mobile comportant les bobinages d'induit et les aimants inducteurs. Nous pouvons remarquer que la structure est totalement tridimensionnelle.

Les principaux avantages de telles structures sont :

- aspect multicellulaire permettant de conserver un bobinage global tout en multipliant le nombre de cellules élémentaires juxtaposées (voir Fig. 3) ;

- concentration de flux aisée par le rapport des surfaces $S_{2} / S_{1}$ (voir Fig. 4), d'où la possibilité d'obtenir des efforts surfaciques importants en utilisant des aimants économiques de type ferrite. 


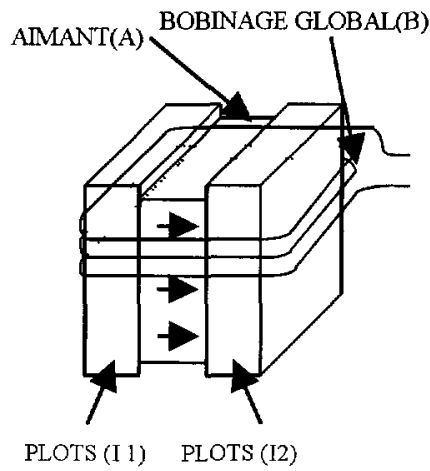

a)
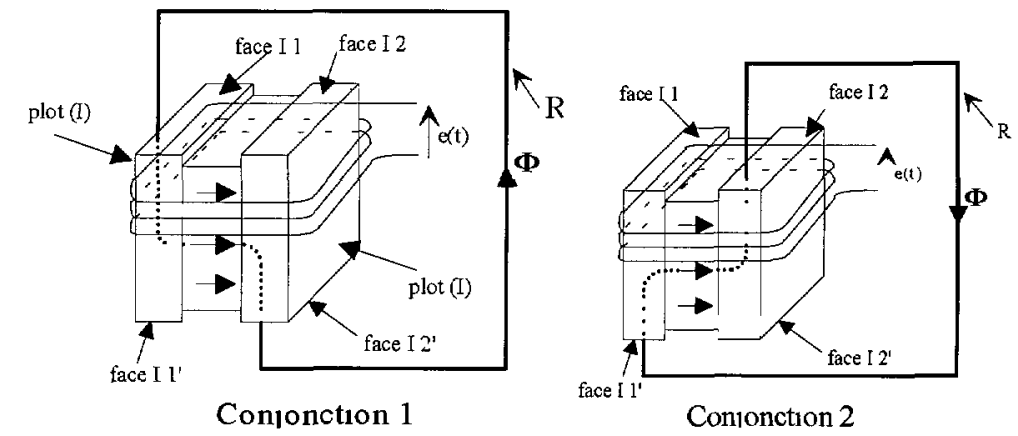

b)

Fig 1 - a) Cellule élémentarre. b) Prncipe de la commutation de flux (positions de conjonction) [a) Elementary cell. b) Flux switching flux principle ]

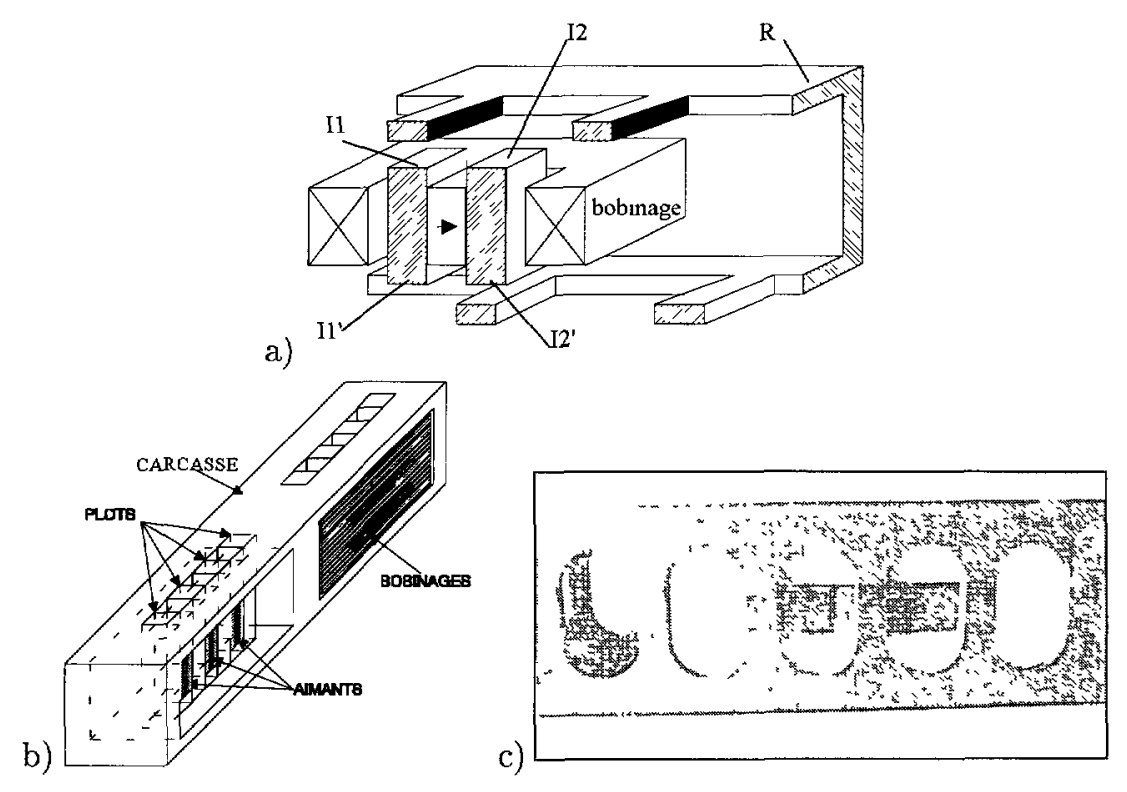

Fig 2. - Actionneur lınéarre réalisé. a) Vue en perspective. b) Partıe mobile comportant deux phases. c) Rail.

[Tested linear actuator. a) Perspectıve view. b) Two phase mobil part. c) Rail ] 


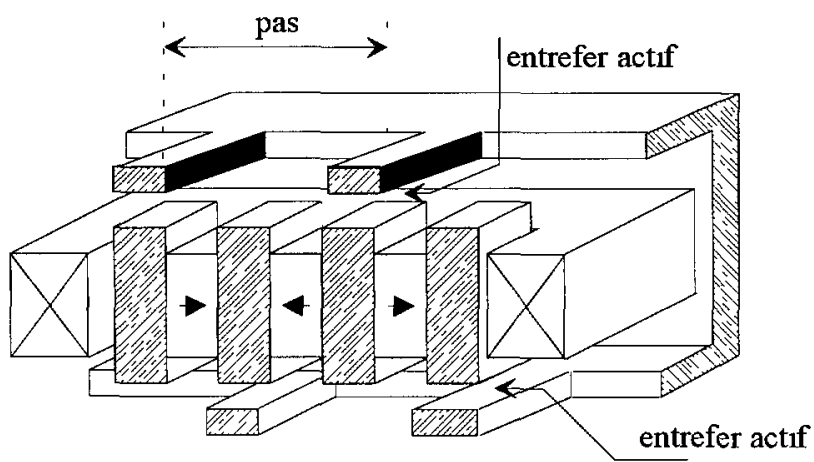

Fig. 3

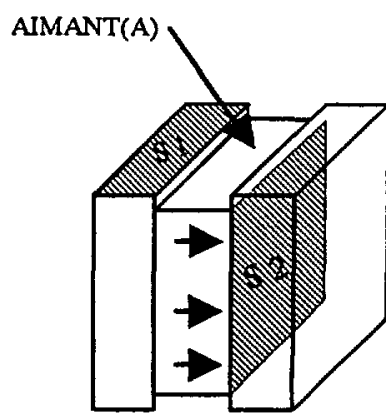

Fig. 4

Fig. 3. - Aspect multıcellulaire de la machine

[Multı-cell topology.]

Fig. 4. - Concentration de flux possible $\left(S_{2}>S_{1}\right)$.

[Flux concentration $\left(S_{2}>S_{1}\right)$ ]

L'optimisation et la modélisation numérique complète de la structure nécessitant un calcul $3 \mathrm{D}$ en régime non linéaire, s'avèrent excessives en temps de calcul et en taille mémoire. Une modélisation utilisant un calcul de champ bidimensionnel par éléments finis relatif à la zone active, associée à une modélisation analytique à partir de réluctances saturables relative aux zones de retours de flux, est présentée Une méthode de détermination des caractéristiques magnétiques de l'actionneur minimisant les points de calcul nécessaires est notamment décrite.

2.2. CyCle Énergétique de Conversion. - La surface du cycle énergétique de conversion représente, dans le plan flux/ampères-tours, l'énergie magnétique convertie. Ce cycle est délimité par les deux caractéristiques magnétiques extrêmes de conjonction (flux maximum des almants à travers le bobinage) et défini par la valeur du courant de l'alımentation considérée ici comme idéale en créneaux. La surface ainsi obtenue correspond donc à l'énergie convertie $\Delta W_{\mathrm{m}}$ sur un pas dentaire (Fig. 3). Il est alors aisé de déterminer l'effort moyen développé sur un pas par la structure à partir de l'équation (1) :

$$
\langle F\rangle=\frac{\Delta W_{\mathrm{m}}}{\text { pas }}
$$

Pour notre part, la notion plus générale de pression tangentielle moyenne (notée $\sigma_{\mathrm{T}}$ ) est utilisée pour calculer et dimensionner nos structures dont l'expression est donnée par (2):

$$
\sigma_{\mathrm{t}}=\frac{\langle F\rangle}{S_{\mathrm{e}}}
$$

où $S_{\mathrm{e}}$ correspond à la surface totale d'entrefer (sur les deux faces).

La figure 5 représente un cycle de fonctionnement réel obtenu par éléments finis. Celui-ci montre la non-linéarité magnétique des caractéristiques magnétiques extrêmes du cycle. Celleci est pénalisante, tant pour les performances intrinsèques des structures comme nous le verrons par la suite que pour la modélisation et les calculs. 


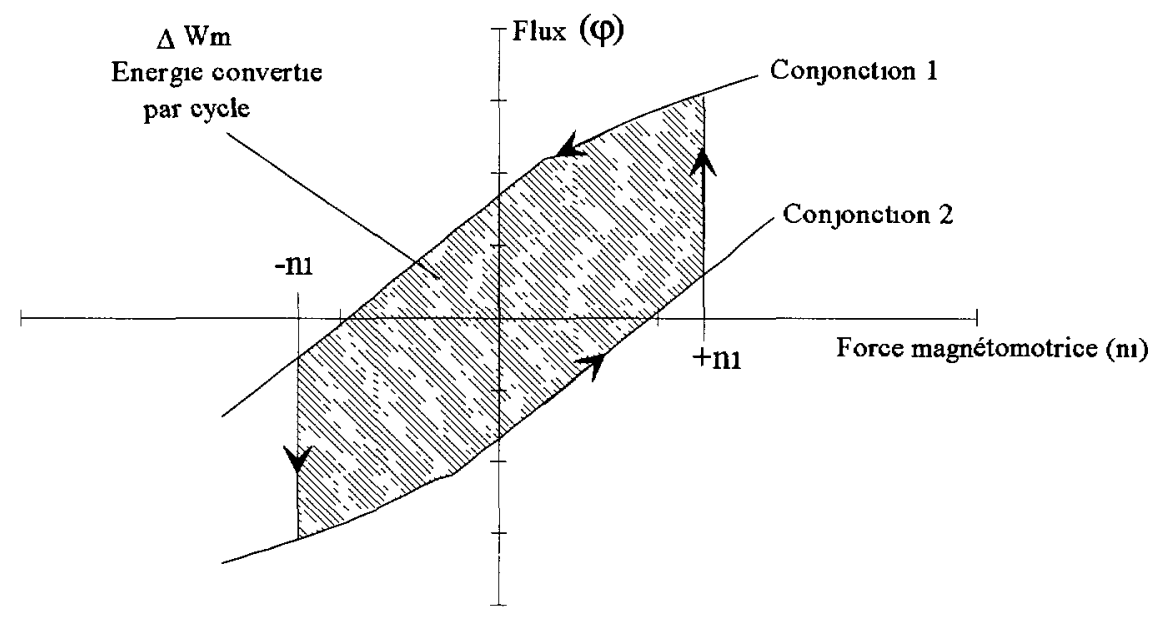

Fıg 5 - Caractéristiques magnétıques extrêmes et cycle énergétique de conversion dans le cas d'une alimentation en créneaux de courant.

[Electromagnetic characteristics in conjunction positions and energetic stroke in ideal square current supply case ]

\section{Modélisation semi-analytique}

Nous détaillerons principalement dans ce chapitre la méthode développée afin de réduire les points de calcul numériques par éléments finis en régime non-linéaire.

3.1. PRINCIPE. - La figure 3 montre l'aspect cellulaire de l'actionneur étudié. La modélisation globale de cet actionneur peut donc se décomposer en différents sous-ensembles (voir Fig. 6) :

- la zone active où se produit la conversion d'énergie magnétique/mécanique, cette zone comprend les $n$ cellules élémentaires identiques et est traversée par la somme des flux actifs de chaque cellule (noté $\varphi$ sur la Fig. 7),

- la région assurant le retour du flux. Cette région est traversée par le flux actif et par le flux de fuites dû au bobinage;

- une zone dans laquelle les fuites se produisent, celle-ci englobe le bobinage.

Ces différentes zones peuvent être représentées sous forme d'un schéma magnétique équivalent (Fig. 7). $V_{\mathrm{b}}$ est la force magnétomotrice du bobinage global appliquée aux $n$ cellules et $R_{3 d}$ est une réluctance liée au circuit de retour tenant compte du changement de section lors d'un passage du flux d'un plan dans un autre. Pour des raisons de précision, le calcul effectué au niveau de la cellule dite élémentaire est numérique (calcul de champ bidimensionnel par éléments finis avec une f.m.m. $n \imath$ ). Dans les autres éléments le calcul est effectué de manière analytique à partir des réluctances saturables.

Cette modélisation est donc basée sur un calcul numérique bidimensionnel. Une correction analytique tridimensionnelle est cependant effectuée à travers l'introduction des réluctances saturables $R_{3 \mathrm{~d}}$. 


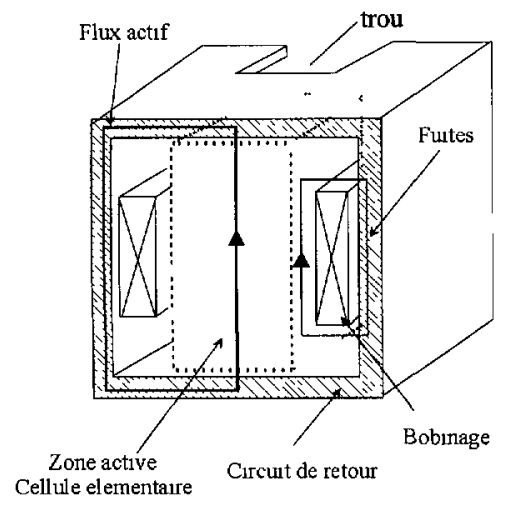

Fig 6

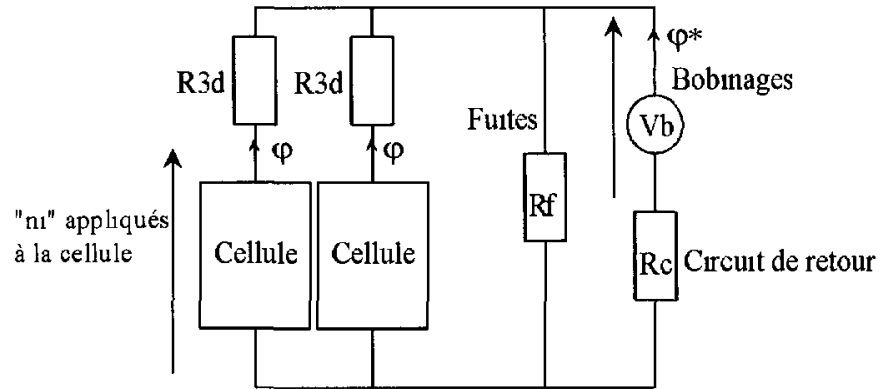

Fig 7

Fig. 6 - Coupe d'un actionneur linéarre rail entourant

[Cross section of linear actuator.]

Fig. 7. - Schéma magnétıque équivalent, cas où la zone active comprend deux cellules élémentarres.

[Equivalent magnetic circuit in two cell case ]

3.2. Modélisation cellulaire. - Pour le calcul de champ par éléments finis, nous devons définir une représentation géométrique simplifiée de la cellule à partir de l'actionneur représenté figure 4. Un motif élémentaire est composé de (voir Fig. 8a) :

- deux aimants permanents d'aimantation opposée ;

- trois pièces ferromagnétiques inter-aimants assurant la concentration de flux ;

- deux entrefers ou jeux mécaniques ,

- trois plots magnétiques décalés les uns par rapports aux autres d'un demı-pas dentaire et représentant la denture du rail ferromagnétique.

À partir du motif élémentaire, nous définissons un motif élémentaire normalisé de hauteur égale à l'unité.

3.2.1. Domaine de calcul normalisé. - Dans le but d'effectuer une analyse paramétrique générale aisément exploitable pour d'autres configurations, notamment polyentrefers, nous définissons des paramètres sans dimension ( $\left.F_{1} g .9 b\right)$ géométriques et électromagnétiques de la façon suivante [12] :

$\lambda=\frac{L_{\mathrm{d}}}{H_{\mathrm{d}}}:$ pas dentaire normalisé,

$a=\frac{H_{\mathrm{a}}}{H_{\mathrm{d}}}:$ hauteur normalisée des aimants,

$e=\frac{E}{H_{\mathrm{d}}}:$ jeu mécanique d'entrefer,

$s=\frac{L_{\mathrm{f}}}{L_{\mathrm{d}}}:$ taux de remplissage des dents,

$t=\frac{L_{\mathrm{a}}}{L_{\mathrm{d}}} \cdot$ taux de remplissage des aimants, 

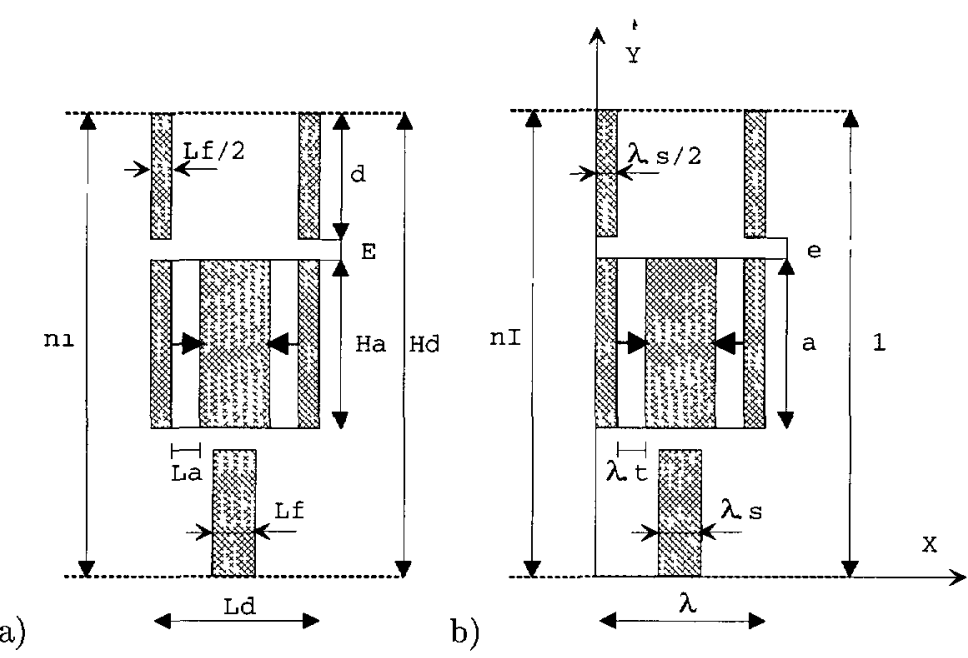

Fig. 8 - Schéma d'un motif élémentaire et définition des paramètres géométriques réels (a) et normalisés (b).

[Elementary pattern and definition of geometrical parameters, (a) real pattern, (b) normalised pattern.]

$n I=\frac{n i}{H_{\mathrm{d}}}:$ force magnétomotrıce normalisée appliquée au domaine $\left(\mathrm{A} \mathrm{m}^{-1}\right)$,

$\left\langle b_{\mathrm{a} 0}\right\rangle=\frac{\left\langle B_{\mathrm{a} 0}\right\rangle}{B_{\mathrm{r}}} \cdot$ induction normalisée moyenne dues aux aimants seuls ( $B_{\mathrm{r}}$ correspond à l'induction rémanente des aimants pour une température de fonctionnement donnée).

3.2.2. Cycle de fonctionnement normalisé. - Le calcul par éléments finis s'effectue sur un motif élémentaire Nous pouvons définir deux positions caractéristiques de la partie mobile appelées positions de conjonction $(\tau=0$ et $\tau=\lambda / 2)$. $\tau$ est la position relative de la partie mobile, la figure $8 \mathrm{~b}$ présente le cas $\tau=0$.

La symétrie des configurations magnétıques dans ces deux positions caractéristiques permet de ne calculer la caractéristıque que pour une seule de ces positions, il est alors possible de déterminer complètement l'énergie convertie par cycle.

En effet, l'effort moyen développé sur un pas d'après (1) s'écrit :

$$
\langle F\rangle=\frac{\Delta W_{\mathrm{m}}}{L_{\mathrm{d}}} .
$$

L'énergie convertie est calculée à partir de la variation de la coénergie magnétique (à courant constant) :

$$
\Delta W_{\mathrm{m}}=\oint \varphi \mathrm{d}(n i)
$$

En utilisant les paramètres réduits définis précédemment, nous obtenons :

$$
\Delta W_{\mathrm{m}}=H_{\mathrm{d}}^{2} \lambda \oint\langle B\rangle \mathrm{d}(n I)
$$

où $\langle B\rangle$ correspond à la moyenne spatiale de l'induction magnétique :

$$
\langle B\rangle=\frac{1}{\lambda} \int_{0}^{\lambda} B_{y}(x, y) \mathrm{d} x .
$$




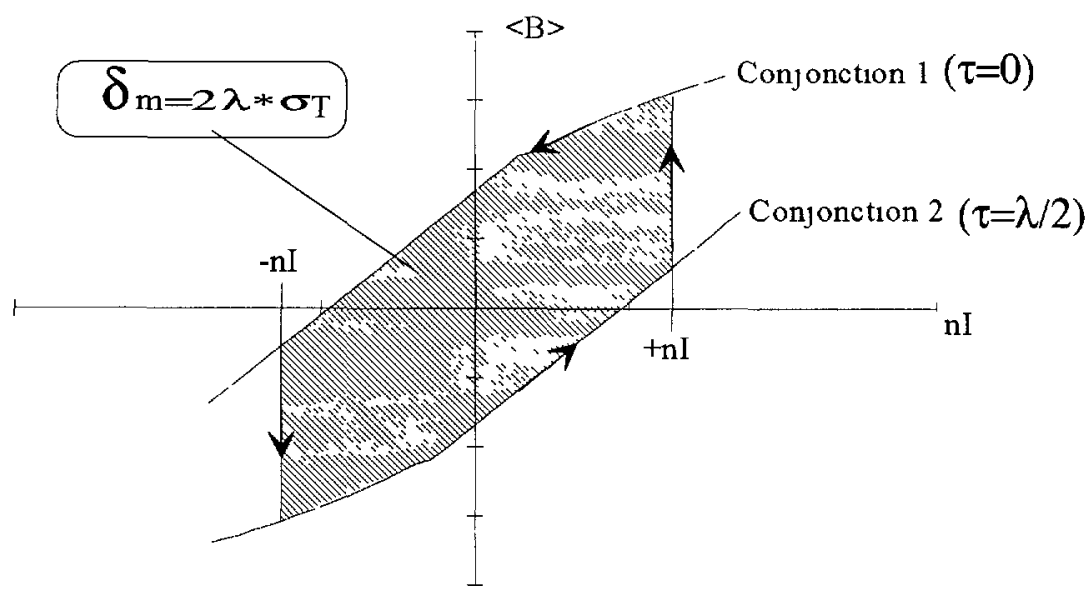

Fig 9 - Aire normalisée du cycle énergétıque de conversion dans le cas d'une alımentation en créneaux de courant.

[Normalised energetic stroke area in 1deal square current supply case ]

Ceci permet alors d'écrire l'équation (2) sous la forme :

$$
\sigma_{\mathrm{T}}=\frac{1}{2 \lambda} \oint\langle B\rangle \mathrm{d}(n I)=\frac{\delta_{\mathrm{m}}}{2 \lambda} .
$$

Cette expression montre qu'au facteur $1 / 2 \lambda$ près, la pression tangentielle moyenne correspond à l'énergie convertie $\delta_{\mathrm{m}}$ dans un cycle représenté dans le plan normalisé $\langle B\rangle(n I)$ (voir Fig. 9). Par définition, en utilisant les paramètres normalisés et pour un domaine de profondeur unité, la perméance incrémentale peut s'écrire :

$$
P=\frac{\Delta \varphi}{\Delta(n i)}=\frac{\Delta(\langle B\rangle)}{\Delta(n I)} \frac{\left(L_{\mathrm{d}}^{*} 1\right)}{H_{\mathrm{d}}}=\lambda \frac{\Delta(\langle B\rangle)}{\Delta(n I)} .
$$

Le rapport $\frac{\Delta(\langle B\rangle)}{\Delta(n I)}$ correspond donc à la perméabilité moyenne incrémentale de la cellule élémentaire que l'on notera par la suite $\langle\mu c\rangle$.

Pour une position de conjonction et en régime linéaire, le point de fonctionnement dans le plan normalisé est décrit par la droite d'équation :

$$
\langle B\rangle=\langle\mu c\rangle n I+\left\langle B_{\mathrm{a}}\right\rangle
$$

où $\left\langle B_{\mathrm{a}}\right\rangle$ correspond à l'induction moyenne due aux aimants seuls (image du flux inducteur) dans la position de conjonction considérée.

En régime saturé, le lieu des points de fonctionnement dans le plan $\langle B\rangle(n I)$ peut être décrit par des segments de droite. Chaque segment d'indice $k$ est régi par une équation de la forme :

$$
\langle B\rangle=\langle\mu c\rangle_{k} n I+\langle B\rangle_{\mathrm{o} k}
$$

où $\langle\mu c\rangle_{k}$ correspond ici à la perméance moyenne incrémentale de la cellule élémentaire. $\langle B\rangle_{\mathrm{o}} k$ représente l'induction moyenne fictıve à vide $(n I=0)$.

Ainsi, le cycle normalisé peut être décrit par un nombre fini de segments de droites. Le calcul des différents segments est effectué par une méthode à pas adaptatif dont le principe est développé dans ce qui suit. 
Tableau I. - Valeurs des constantes intervenant dans l'expression de l'équation de la perméabilité.

[Coefficient values of analytical model of relative permeability.]

\begin{tabular}{|c|c|c|c|c|}
\hline$\mu_{r_{0}}$ & $B_{\mathrm{s}}$ & $g$ & $b$ & $H_{\mathrm{c}}$ \\
\hline 920 & 1.6 & 1.2 & $8 \times 10^{-4}$ & 2000 \\
\hline
\end{tabular}

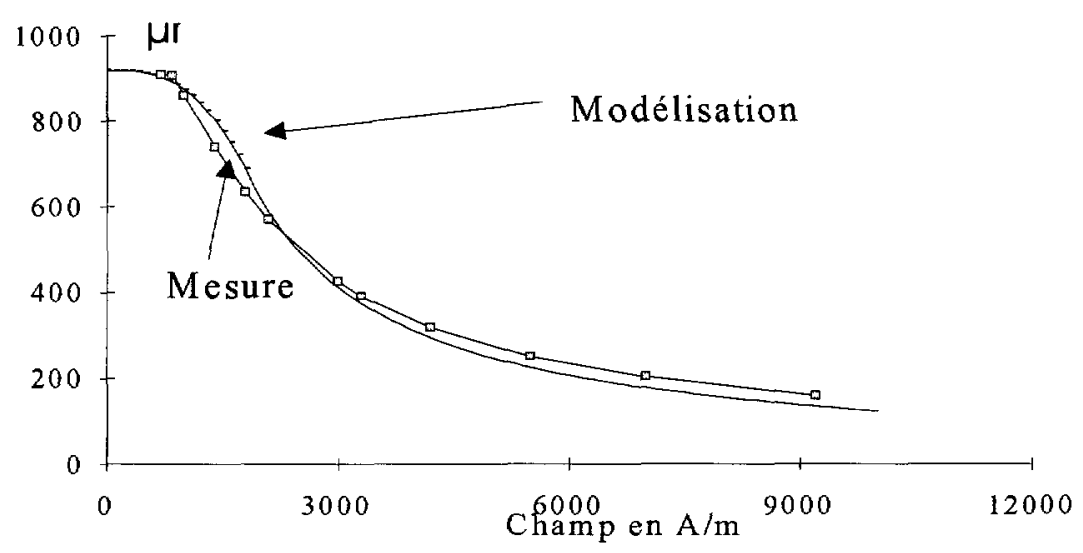

Fıg. 10. - Comparaıson de la perméabilité relatıve mesurée $(1 \mathrm{~Hz})$ et modélisée.

[Comparison of the measure and analytical modelised relative permeability]

3.2.3. Méthode de calcul du cycle de fonctionnement. - Du fait de la non linéarité des caractéristiques magnétiques des matériaux ferromagnétiques utilisés dans les prototypes réalisés et de l'utilisation de pièces inter-aimants concentratrices de flux, la prise en compte de la variation de la perméabilité en fonction du champ magnétique s'avère nécessaire. Cette caractéristique magnétique est décrite, dans notre cas, par deux équations analytiques [11] :

$$
\begin{cases}\mu_{\mathrm{r}}(H)=\mu_{\mathrm{r}_{0}} \mathrm{e}^{\left[-\frac{g}{3}\left(\frac{b H}{B_{\mathrm{S}}}\right)^{3}\right]} & \text { pour } H \leq H_{\mathrm{c}} \\ \mu_{\mathrm{r}}(H)=1+\frac{\mu_{\mathrm{r}_{0}} \mathrm{e}^{\left[-\frac{g}{3}\right]}-1}{\left(b H / B_{\mathrm{S}}\right)} & \text { pour } H>H_{\mathrm{c}} .\end{cases}
$$

Les coefficients correspondent dans le cas étudié aux valeurs données dans le tableau I.

La figure 10 montre la caractéristique mesurée de la perméabilité du matériau utilisé (XC10, fréquence $1 \mathrm{~Hz}$ ) comparée à la modélisation analytique introduite dans les calculs.

La détermination des caractéristiques magnétiques délimitant le cycle de fonctionnement pour une alimentation en créneaux de courant dépend du nombre de points de calcul $(\langle B\rangle, n I)$. Pour une géométrie présentant de fortes saturations locales et globales, les caractéristıques magnétiques sont complexes. Leur calcul impose donc un nombre de point $(\langle B\rangle, n I)$ important De plus, la limite de conversion et donc la plage de variation de la force-magnétomotrice (f.m.m.) pour une géométrie donnée sont a prıor indéterminées Pour pallier à ces inconvénients, nous avons développé une méthode, dite à pas adaptatif, permettant de définir complètement les caractéristiques magnétiques extrêmes du cycle de fonctionnement normalisé à partir d'un nombre de point minimal.

Pour faciliter la compréhension de la méthode, nous présentons uniquement le déroulement des calculs pour le premier quadrant du plan $\langle B\rangle(n I)$. 


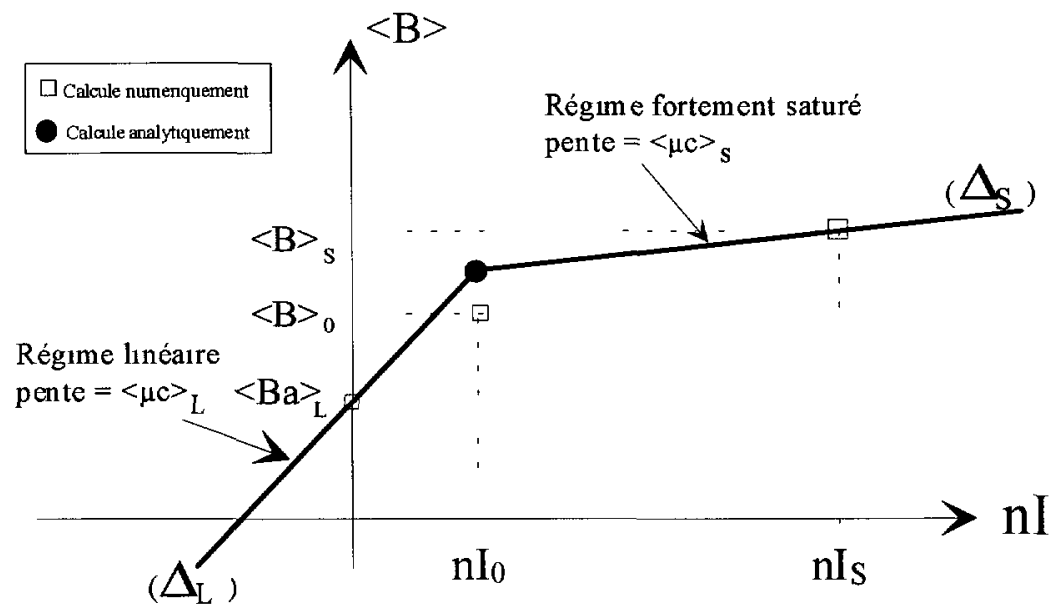

Fıg. 11. - Asymptotes de la caractéristıques magnétique extrême en positıon de conjonction

[Asymptote of magnetic characteristics in conjunction position.]

Tout d'abord, nous définissons deux asymptotes (voir Fig. 11) :

La première asymptote $\left(\Delta_{\mathrm{S}}\right)$ correspond à un régime fortement saturé. L'équation de celle-ci est donnée par :

$$
\langle B\rangle=\langle\mu c\rangle_{\mathrm{s}}\left(n I-n I_{\mathrm{s}}\right)+\langle B\rangle_{\mathrm{s}}
$$

où $\langle\mu c\rangle_{s}$ correspond à la perméabilité relative moyenne incrémentale du domaine en régime fortement saturé, sa valeur vaut donc $\mu_{0}$.

$\langle B\rangle_{\mathrm{s}}$ est l'induction moyenne (ou le flux par unité de surface) correspondant au courant $n I_{\mathrm{s}}$. La valeur de ce dernier, compte tenue de la valeur imposée de $\langle\mu c\rangle_{s}$, est directement liée à l'induction de saturation $B_{\mathrm{s}}$ du matériau magnétique utilisé :

$$
n I_{\mathrm{s}}=\frac{B_{\mathrm{s}}}{\mu_{0}}
$$

La seconde asymptote $\left(\Delta_{\mathrm{L}}\right)$ correspond au régime linéaire L'équation de celle-ci est donnée par :

$$
\langle B\rangle=\langle\mu c\rangle_{\mathrm{L}} n I+\left\langle B_{\mathrm{a}}\right\rangle_{\mathrm{L}}
$$

où $\langle\mu c\rangle_{\mathrm{L}}$ correspond à la perméabılité moyenne du domaine en régime lınéaire et $\left\langle B_{\mathrm{a}}\right\rangle_{\mathrm{L}}$ représente l'induction moyenne à vide $(n I=0)$, calculés par éléments finis en régime linéaıre.

La caractéristique recherchée est donc située au-dessous des deux asymptotes $\left(\Delta_{\mathrm{S}}\right)$ et $\left(\Delta_{\mathrm{L}}\right)$. La méthode consiste alors à définir la caractéristique par des segments de droites calculés séquentiellement à partir des deux asymptotes

Le nombre de ces segments est fonction de :

- la précision souhaitée;

- la présence plus ou moins marquée de saturations locales.

Le choix des points de calcul $\left(\langle B\rangle_{k}, n I_{k}\right)$ est effectué à partir des courants $n I_{k}$ déterminés par constructions géométriques successives. Les inductions moyennes $\langle B\rangle_{k}$ correspondantes sont calculées par Éléments Finis en régime non linéaire (E.F.n.l.). 


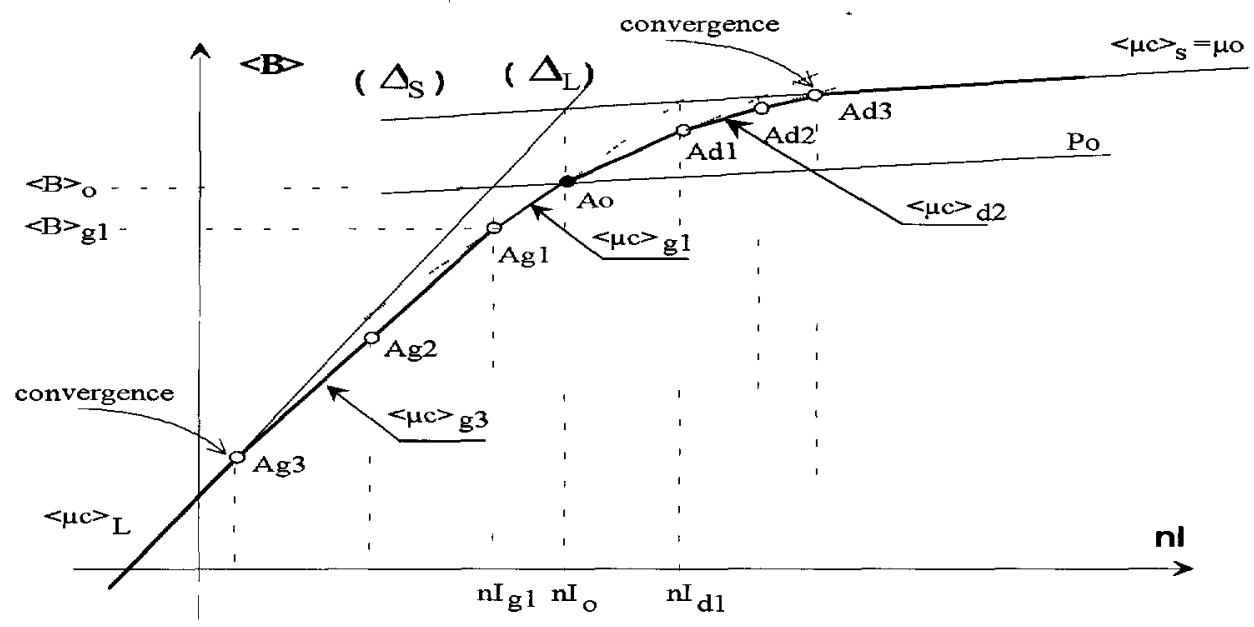

Fig. 12. - Description de la méthode du pas adaptatıf.

[Adaptive method principle.]

L'intersection de $\left(\Delta_{\mathrm{s}}\right)$ et $\left(\Delta_{\mathrm{L}}\right)$ impose le premier point de calcul correspondant au courant $n I_{0}$ défini par (13). La valeur de $\langle B\rangle_{0}$ correspondante est alors calculée par E.F.n.l. :

$$
n I_{0}=\frac{\left(\left\langle B_{\mathrm{a}}\right\rangle_{\mathrm{L}}-\langle B\rangle_{\mathrm{s}}\right)+\langle\mu c\rangle_{\mathrm{s}} n I_{\mathrm{s}}}{\langle\mu c\rangle_{\mathrm{s}}-\langle\mu c\rangle_{\mathrm{L}}}
$$

Le point $A_{0}\left(\langle B\rangle_{0}, n I_{0}\right)$ sépare la caractéristique recherchée en deux domaines gauche (indice g) et droite (indice d) tel que schématisé par la figure 12 .

L'intersection de $\left(\Delta_{\mathrm{L}}\right)$ avec la droite de pente $\langle\mu c\rangle_{\mathrm{s}}$ passant par le point $\mathrm{A}_{0}$ définit le courant $n I_{\mathrm{g} 1}$ pour lequel le calcul par E.F.n.l. donne le point $\mathrm{A}_{\mathrm{g} 1}\left(\langle B\rangle_{\mathrm{g} 1}, n I_{\mathrm{g} 1}\right)$ avec :

$$
n I_{\mathrm{g} 1}=\frac{\left(\left\langle B_{\mathrm{a}}\right\rangle_{\mathrm{L}}-\langle B\rangle_{0}\right)+\langle\mu c\rangle_{\mathrm{s}} n I_{0}}{\langle\mu c\rangle_{\mathrm{s}}-\langle\mu c\rangle_{\mathrm{L}}}
$$

La droite passant par $\mathrm{A}_{0}$ et $\mathrm{A}_{\mathrm{g} 1}$ possède la pente $\langle\mu c\rangle_{\mathrm{g} 1}$ définie par ·

$$
\langle\mu c\rangle_{\mathrm{g} 1}=\frac{\left(\langle B\rangle_{0}-\langle B\rangle_{\mathrm{g} 1}\right)}{n I_{0}-n I_{\mathrm{g} 1}}
$$

D'où on peut généraliser le calcul pour le $k$-ième point par .

$$
\langle\mu c\rangle_{\mathrm{g} k}=\frac{\left(\langle B\rangle_{\mathrm{g}(k-1)}-\langle B\rangle_{\mathrm{g} k}\right)}{n I_{\mathrm{g}(k-1)}-n I_{\mathrm{g} k}} .
$$

Ce calcul est effectué par itération jusqu'à ce que le critère de convergence (Éq. (17)) soit atteint :

$$
\left|\frac{\langle\mu c\rangle_{\mathrm{g} k}-\langle\mu c\rangle_{\mathrm{L}}}{\langle\mu c\rangle_{\mathrm{L}}}\right| \leq \epsilon \quad \forall \quad n I \in\left[0, n I_{0}\right] .
$$

La même méthode est ensuite appliquée à la partie droite de la caractéristique jusqu'au critère de convergence :

$$
\left|\frac{\langle\mu c\rangle_{\mathrm{d} k}-\mu_{0}}{\mu_{0}}\right| \leq \epsilon \quad \forall \quad n I \in\left[n I_{0}, n I_{\mathrm{S}}\right] .
$$




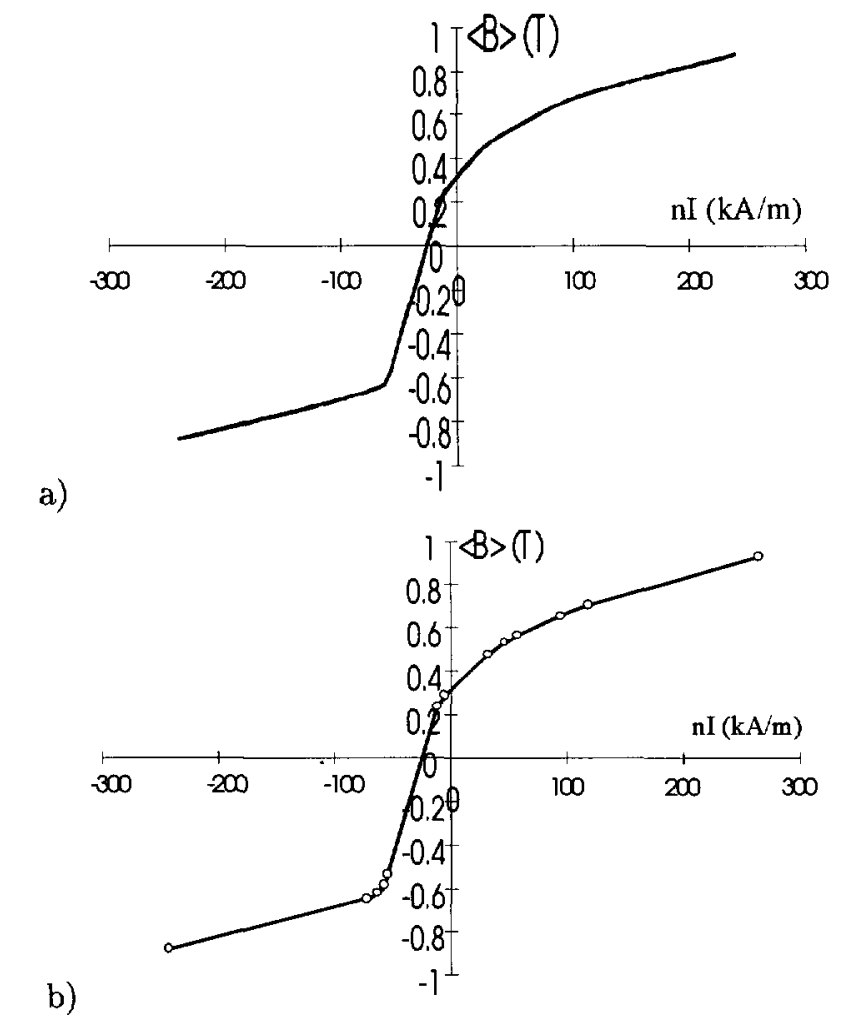

Fig. 13. - Caractéristıques calculées par éléments finıs en régime non linéaire a) Méthode à pas constant (100 points), b) méthode à pas adaptatif (20 points) pour $\lambda=0,4, s=0,3, e=0,01, t=0,3$ et $a=0,7$.

[Non-linear magnetic characteristic computed using f.e. method. a) constant step method (100 points), b) adaptive method (20 points)] for $\lambda=0.4, s=03, e=0.01, t=03, a=0.7$.]

Pour les ampères-tours négatifs ( $2^{\mathrm{e}}$ et $3^{\mathrm{e}}$ quadrants) le principe reste identique.

La figure 13 compare les méthodes à pas constant (100 points) et à pas adaptatif. La précision obtenue est identique. Grâce à la méthode du pas adaptatif, une caractéristique complète est décrite fidèlement avec un nombre minimal de points (environ 20 points) quelle que soit la configuration de notre modèle. Les avantages de la méthode proposée sont donc multiples, recherche automatique des limites extrêmes des courants et augmentation de la densité de points dans les régions fortement non-linéaires (coudes de saturation).

3.3. ÉTUDE PARAMÉTRIQUE INTRINSÈQUE. - La méthode décrite s'avérant suffisamment rapide, nous avons pu effectuer une étude paramétrique intrinsèque relative aux performances de la cellule élémentaires. Les contraintes de fonctionnement telles que l'alimentation ou les courants de Foucault ne sont pas prises en compte dans cette étude.

Nous avons donc calculé la pression tangentielle moyenne pour diverses configurations géométriques correspondant à la variation de 5 paramètres $(\lambda, a, s, t, e)$, et ceci uniquement pour des aimants de type ferrite pour lesquels nous avons considéré leurs caractéristiques à $80^{\circ} \mathrm{C}$ où $B_{\mathrm{r}}=0,35 \mathrm{~T}$ (voir annexe). 


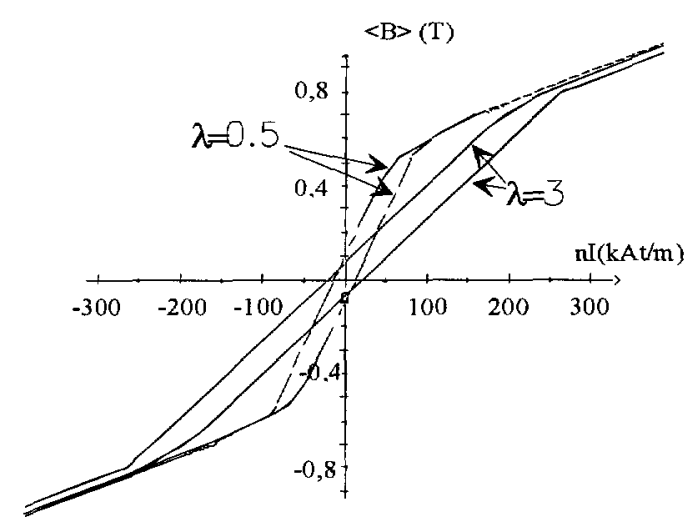

a)

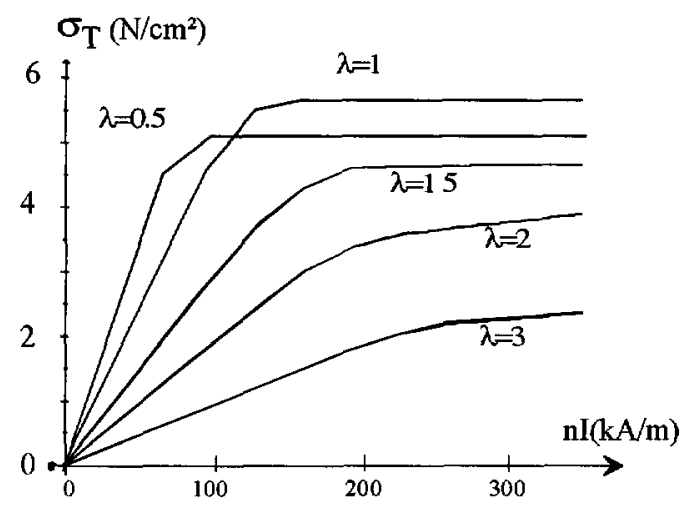

b)

Fig. 14. - Influence du pas dentaire normalisé sur les caractéristıques magnétiques de conjonction (a) et la pression tangentielle moyenne (b) pour $s=0,3, e=0,04, t=0,3$ et $a=0,5$.

[Influence of the normalised tooth pitch on the magnetic characteristics in conjunction position (a) and on the magnetic shear stress (b) for $s=0.3, e=0.04, t=0.3, a=0.5$ ]

3.4. InfluENCE DU PAS DENTAIRE $(\lambda)$. - En régime linéaire, la diminution du pas dentaire normalisé $(\lambda)$ n'entraîne pas une décroissance majeure de l'énergie magnétique convertie (voir Fig. 14a). Par conséquent, sachant que l'effort surfacique $\left(\sigma_{\mathrm{T}}\right)$ est inversement proportionnel au pas (voir relation (7)), la diminution du pas engendre l'augmentation de $\sigma_{\mathrm{T}}$.

En régime non-linéaire, les saturations des pièces ferromagnétiques concentratrices de flux engendrent des fuites magnétiques. Ces fuites sont d'autant plus importantes que le pas est faible. La diminution du pas ne conserve donc pas l'énergie convertie en régime non-linéaire et de ce fait il y a apparition d'une valeur optimale du pas. Cette dernière est fonction du niveau de saturation et donc de la valeur de la force-magnétomotrice appliquée.

Pour visualiser ce phénomène, nous avons tracé les caractéristiques flux/ampères-tours correspondant à deux valeurs de $\lambda$ différentes (Fig. 14), puis les caractéristiques efforts surfaciques en fonction du courant pour différents $\lambda$ (Fig. 15). Celles-ci montrent bien la limite du courant utile. Pour le cas représenté avec $\lambda=1$, la limite de conversion se situe à $150 \mathrm{kA} \mathrm{m}^{-1}$. Alors que pour $\lambda=0,5$ cette limite est d'environ $70 \mathrm{kA} \mathrm{m}^{-1}$. Au-delà, l'énergie convertie devient négligeable.

La figure 15 représente pour une configuration l'effort tangentiel par unité de surface en fonction du pas dentaire normalisé pour trois valeurs de courant correspondant à un régime linéaire $\left(50 \mathrm{kA} \mathrm{m}^{-1}\right)$, faiblement $\left(100 \mathrm{kA} \mathrm{m}^{-1}\right)$ puis fortement saturé $\left(300 \mathrm{kA} \mathrm{m}^{-1}\right)$.

Cette figure montre notamment un optimum pour $\lambda \approx 1$ et pour une force magnétomotrice de $300 \mathrm{kA} \mathrm{m}^{-1}$, alors que pour des courants plus faibles, l'optimum correspond à des valeurs de pas plus faibles.

Il est à noter qu'il est possible d'obtenir une valeur de pression moyenne intrinsèque importante, de plus de $5 \mathrm{~N} \mathrm{~cm}^{-2}$, avec une structure optimisée Cependant, cette valeur ne prend pas en compte la limite de désaimantation. Celle-ci sera étudiée par la suite.

3.4.1. Influence du taux de remplissage des dents (s). - Le taux de remplissage des dents est un paramètre qui est généralement adaptable dans la conception d'une machine à bobinage global sans influence sur les pertes Joule. Nous allons donc analyser son influence sur la valeur de l'effort surfacique. 


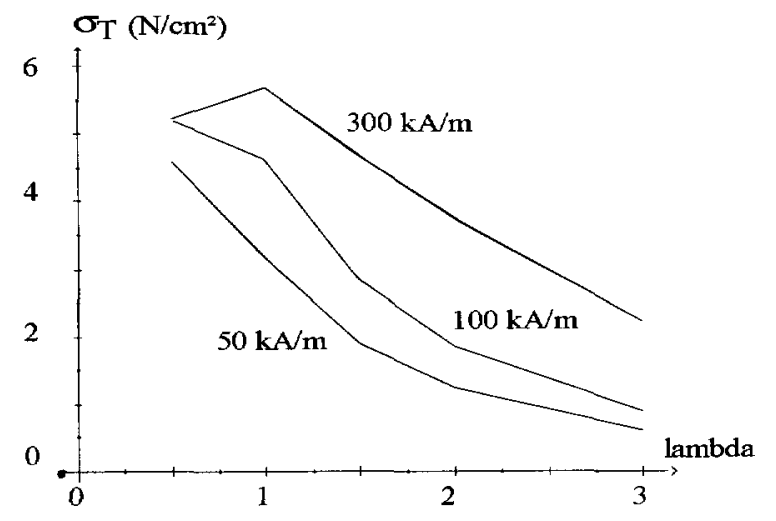

Fig 15. - Influence du pas dentaire normalısé sur la pression tangentielle moyenne $\sigma_{\mathrm{T}}$ pour $s=0,3$, $e=0,04, t=0,3$ et $a=0,5$

[Influence of the normalised tooth pitch on the the magnetic shear stress for $s=0.3, e=0.04, t=0.3$, $a=0.5$.]

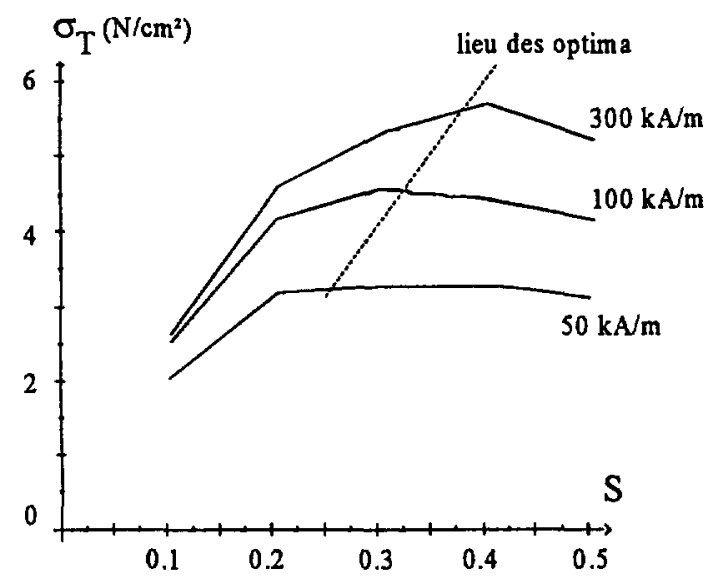

Fig. 16. - Influence du taux de remplissage des dents sur $\sigma_{\mathrm{T}}$ pour $\lambda=1, e=0,04, t=0,3$ et $a \doteq 0,6$. [Influence of the normalised teeth width on the the stress factor for $\lambda=1, e=0.04, t=0.3, a=06$.]

En régime linéaire, l'optimum de $s$ est de l'ordre de 0,3 [9]. Le phénomène de saturation modifie cette valeur et augmente celle-ci afin de fournir une section de passage de flux plus importante comme le montre la figure 16 .

Le taux de remplissage des dents optimal est donc un paramètre fortement dépendant du niveau de saturation (valeur de $n I$ ). Nous verrons par la suite que les configurations optımales nécessitent une valeur de $s$ comprise entre 0,3 et 0,5 . Ceci, dans le cas de l'actionneur étudié où le rail entoure la partie mobile, correspond à une surface "trouée" de $50 \%(s=0,5)$ et de $70 \%(s=0,3)$ de la surface totale supérieure et inférieure du tube.

Remarque - Les valeurs de $s$ supérieures à 0,5 n'ont pas encore été étudiées et devront l'être par la suite pour tenir compte d'un éventuel fonctionnement en mode monophasé [7]. 

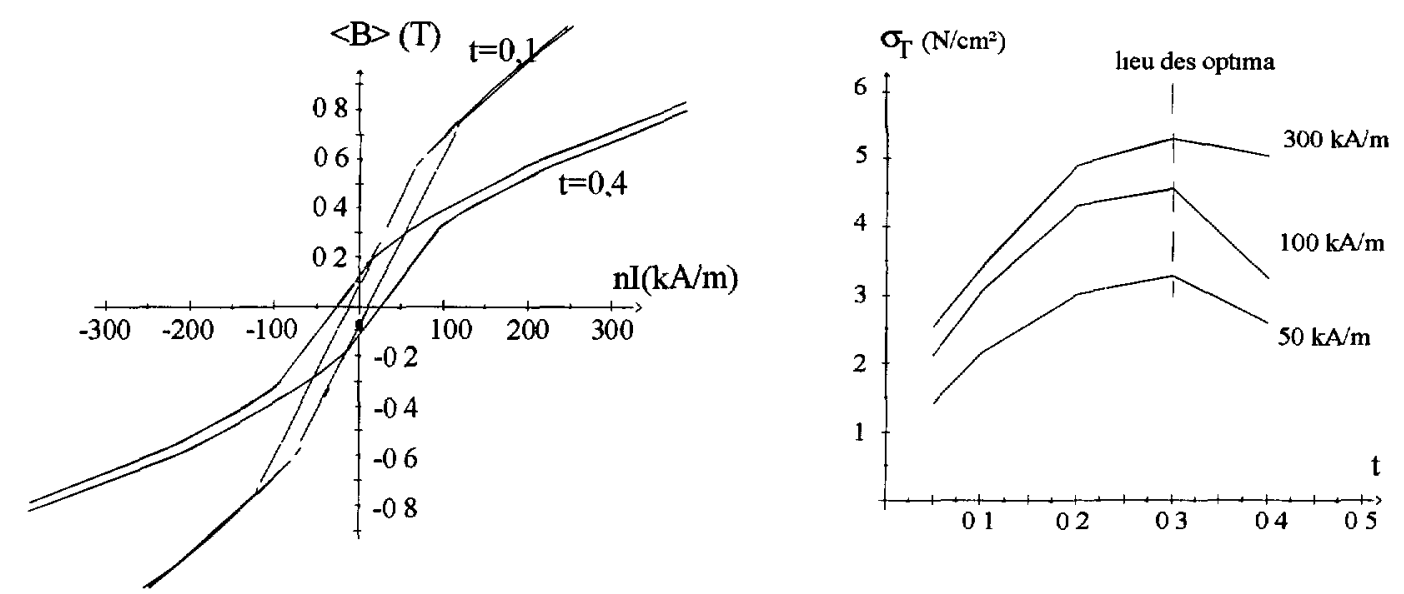

a)

b)

Fig 17. - Influence du taux de remplissage des aimants sur les caractéristiques magnétiques (a) et la pression tangentielle moyenne (b) pour $\lambda=1, e=0,04, s=0,3$ et $a=0,6$.

[Influence of the normalised magnet's width on the magnetic characteristics (a) and on the magnetic shear stress (b) for $\lambda=1, e=0.04, s=0.3, a=0.6$.]

3.4.2. Influence du taux de remplissage des aimants $(t)$. - Le taux de remplissage des aimants détermine notamment, pour une configuration donnée, la section de passage de flux dans les plots. Il influence la "concentration du flux de l'aimant" à la sortie des plots, son rôle est déterminant sur le niveau de saturation de la cellule et conditionne directement le volume d'aimant. D'autre part, ce phénomène dépend de l'induction rémanente $B_{\mathrm{r}}$.

Les résultats de calculs représentés par la figure 17 montrent que le taux de remplissage des aimants maximisant l'effort par unité de surface avec des aimants ferrites est de 0,3. De plus, ce paramètre conditionne de manière spécifique l'amplitude du champ magnétique dans l'aimant dû à la réaction magnétique d'induit et par conséquent la limite de désaimantation que nous aborderons ultérieurement.

3.4.3. Influence de la hauteur d'aimant (a).-- Comme pour le taux de remplissage des aimants $t$, leur hauteur normalisée conditionne principalement la concentration de flux et le volume d'aimant nécessaire. En régime linéaire, la valeur de $a$ maximisant l'effort par unité de surface est comprise entre 0,6 et 0,7 [9]. En effet, pour des valeurs plus faibles, la section utile des aimants est amoindrie et pour des valeurs plus importantes, le court-circuit des aimants est accru.

La courbe donnée sur la figure 18a montre que les optima de $a$ se déplacent d'une manière assez sensible en fonction du courant ( $a=0,7$ en régime linéaire et $a=0,5$ en régime fortement saturé). La saturation limite donc l'intérêt d'utiliser des hauteurs d'aimant importantes. Dans ce dernier cas, la saturation accroît fortement le court-circuit des aimants déjà constaté en régime linéaire comme montré par la figure $18 \mathrm{~b}$.

3.4.4. Influence de l'entrefer (e). - Afin d'évaluer les performances limites de la cellule en terme de poussée, nous avons effectué un calcul de la pression moyenne pour un cas théorıque où $e=0$. Dans ce cas, les résultats ont montré une valeur de $7,8 \mathrm{~N} \mathrm{~cm}^{-2}$ en régime saturé. L'entrefer augmentant, $\sigma_{\mathrm{T}}$ décroît de manière quasi linéaire en régime fortement saturé. Ainsi, 


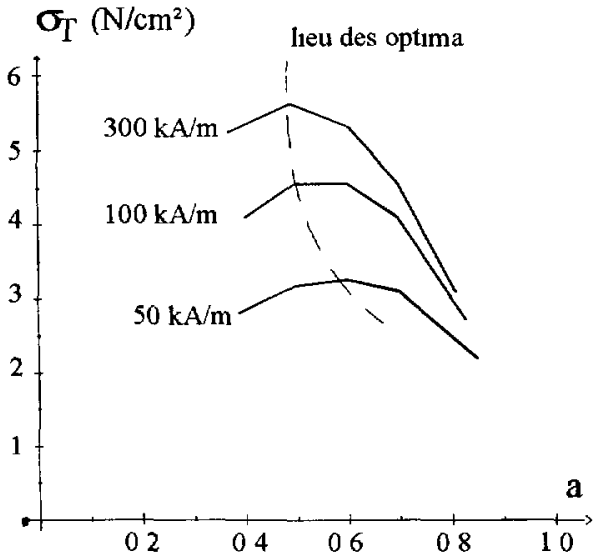

a)

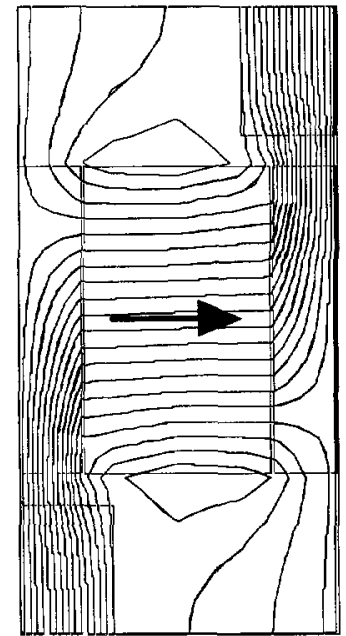

b) $a=0,5$

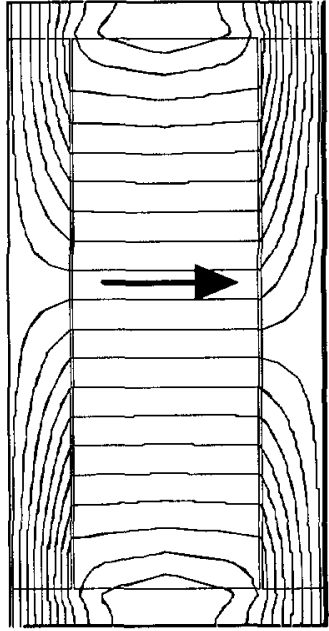

$a=0,9$

Fig 18. - a) Influence du taux de remplissage des aimants sur la pression tangentielle moyenne $\sigma_{\mathrm{t}}$ pour $\lambda=1, e=0,04, s=0,3$ et $t=0,3$. b) Cartes de champ inducteur en position de conjonction pour deux hauteurs d'aimants pour $\lambda=1, e=0,04, s=0,3, t=0,3$.

(a) Influence of the normalısed magnet's heigth on the magnetic shear stress (b) for $\lambda=1, e=0.04$, $s=0.3, t=0.3$. b) Magnet induction distribution for two values of magnet heigth for $\lambda=1, e=0.04$, $s=0.3, t=0.3$.]

pour $e=0,08, \sigma_{\mathrm{T}}$ vaut $3,8 \mathrm{~N} \mathrm{~cm}^{-2}$ soit une décroissance d'environ $50 \%$. Cette décroissance est due essentiellement au court-circuit des aimants à travers le jeu mécanique comme le montre la figure 19a.

L'entrefer est un paramètre fondamental très influent mais il est généralement fixé par le cahier des charges et correspond à la limite mécanique de réalisation, celui-ci devra être, du point de vue électromagnétique, le plus petit possible. On notera cependant (voir Fig. 19b), que l'influence de $e$ est moins importante en régime saturé qu'en linéaire. D'autre part, on montre [5] que cette influence est tributaire du facteur de concentration de flux $\xi$, dont l'expression est donnée par la relation (19)

$$
\xi=\frac{2 a}{\lambda s}
$$

3.4.5. Abaque des optima. - Pour chaque valeur d'entrefer et pour toutes les combinaisons des autres paramètres $(\lambda, a, s, t$ et $n I)$ un calcul de la pression tangentielle moyenne $\left(\sigma_{\mathrm{T}}\right)$ a été effectué grâce au modèle developpé. Les résultats ont constitué une base de donnée à partir de laquelle nous avons recherché, pour chaque courant, les configurations optimales maximisant $\sigma_{\mathrm{T}}$ (noté alors $\sigma_{\mathrm{T}_{\mathrm{OPT}}}$ ). Les résultats obtenus peuvent être présentés sous forme d'abaque.

La figure 20 représente l'abaque pour une valeur d'entrefer de 0,04. Sur la même figure, nous avons également représenté la variation du coefficient optimal (noté $\xi_{\mathrm{OPT}}$ ) de concentration de flux.

Les résultats montrent notamment que :

- la pression moyenne limite obtenue pour $e=0,04$ est d'environ $6 \mathrm{~N} \mathrm{~cm}^{-2}$ pour un fonctionnement en limite de désaimantation. Pour cette valeur, la configuration optimale correspondante est : $\lambda=1, a=0,5, s=0,3$ et $t=0,3$; 


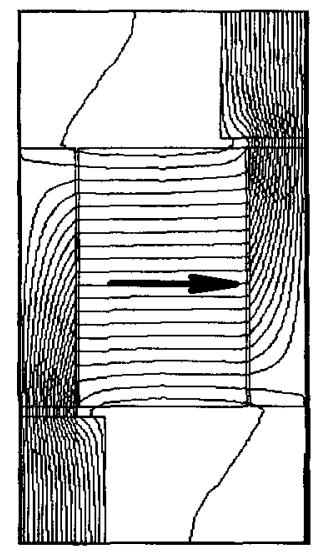

a) $e=0,01$

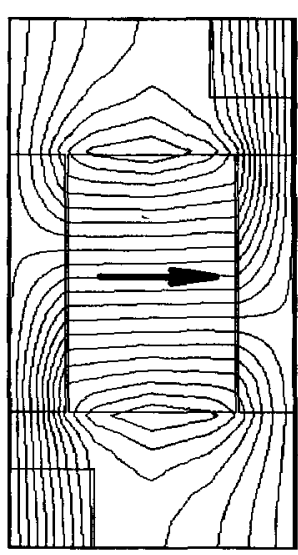

$e=0,1$

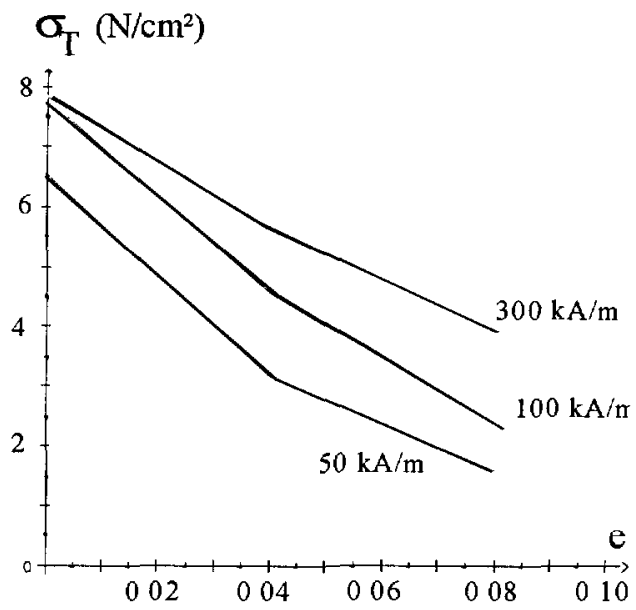

b)

Fig. 19 - a) Cartes de champ inducteur en position de conjonction pour deux valeurs d'entrefer relatif pour $\lambda=1, a=0,5, s=0,3, t=0,3$. b) Influence du jeu mécanique réduit sur $\sigma_{\mathrm{T}}$ pour $\lambda=1$, $a=0,5, s=0,3,=0,3$

[a) Magnet induction distribution for two values of normalised air-gap for $\lambda=1, a=0.5, s=0.3$, $t=03$. b) Influence of the normalised arr-gap on the stress factor for $\lambda=1, a=0.5, s=0.3, t=0.3$.]

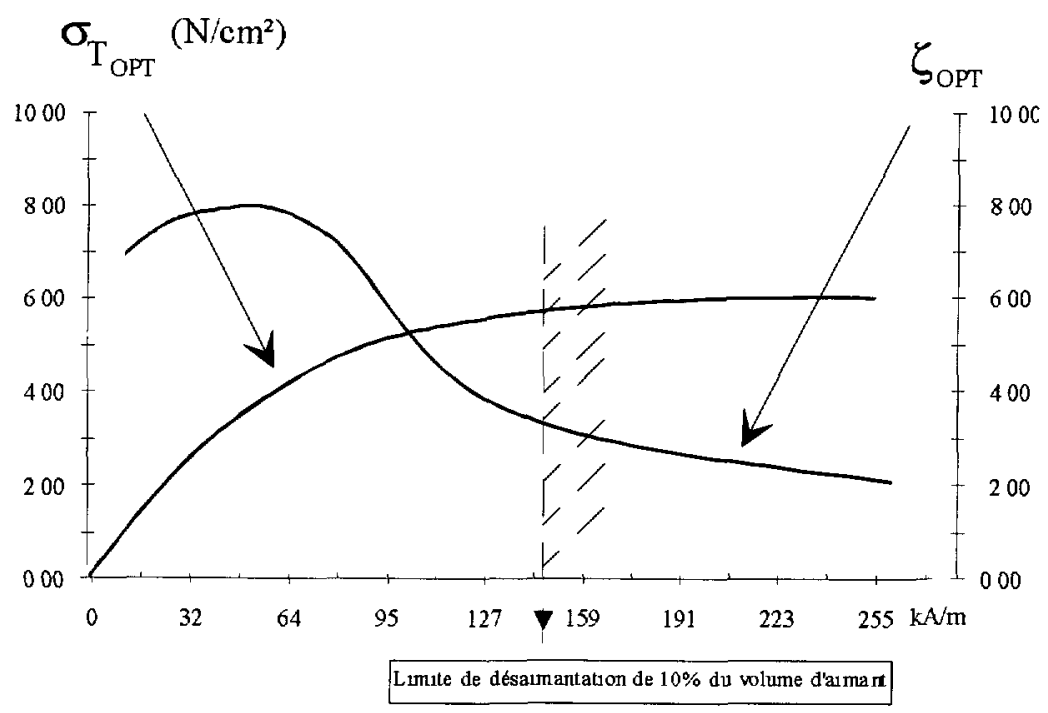

Fig. 20 - Performances optimales intrinsèques pour un entrefer normalisé de 0,04 et $B_{\mathrm{r}}=0,35 \mathrm{~T}$ [Optimal performances for a normalised air-gap $e=004$ and $B_{\mathrm{r}}=0.35 \mathrm{~T}$ ] 
- l'obtention de pressions moyennes élevées nécessite une forte concentration du flux (rapport voisin de 8 dans le cas d'aimants ferrites). À l'inverse, en régime saturé, le rapport de concentration devra être faible, de l'ordre de 3.

L'abaque présenté correspond aux performances intrinsèques. Ces performances sont limitées par différentes contraintes. Une limite importante est relative à la désaimantation. L'introduction simplifiée de cette limite est effectuée pour chacune des configurations optimales par un calcul du champ magnétique dans l'aimant en régime non-linéaire. Le principe consiste à calculer les composantes du champ de réaction d'induit dans l'aimant suivant l'axe d'aimantation des aimants (axe $\mathrm{O} X^{\prime}$ ) pour la position de conjonction.

La répartition de cette composante du champ dans l'aimant est comparée à la valeur du champ de désaimantation admissible par l'aimant noté $H_{k}$. La figure 21 représente cette répartition.

Nous avons calculé la variation de la moyenne volumique du champ suivant l'axe d'aimantation $\left(O X^{\prime}\right)$ dans l'aimant en fonction de la force magnétomotrice appliquée (voir exemple Fig. 21a).

Ces résultats montrent que le phénomène de saturation des pièces ferromagnétiques diminue la perméabilité moyenne du domaine élémentaire et par conséquent repousse la limite du courant démagnétisant. La saturation est alors favorable à la "protection" des aimants.

On note cependant que cette répartition du champ démagnétisant est fortement non homogène dans l'aimant. Il s'agit alors d'évaluer les zones de l'aimant les plus contraintes en termes de désaimantation. Une représentation tridimensionnelle permet de visualiser ces zones.

Ainsi, à titre d'exemple, le cas représenté sur la figure $21 \mathrm{~b}$ montre une induction moyenne suivant l'axe $X^{\prime}$ de $0,26 \mathrm{~T}$ inférieure au champ de désaimantation $\mu_{0} H_{k}$ alors que les zones proches des points $\mathrm{B}$ et $\mathrm{D}$ sont désaimantées. Cependant, le volume d'aimant concerné reste faible. Nous considérons que la désaimantation globale ne sera atteinte que lorsqu'une certaine proportion du volume d'aimant (notée $V_{\mathrm{d}}$ ) est désaimantée. La limite de désaimantation représentée sur l'abaque figure 20 correspond à $V_{\mathrm{d}}=10 \%$.

3.4.6. Performances globales de l'actionneur. - Les performances globales sont déduites des performances de la cellule élémentaire présentée précédemment. Ce calcul est effectué en utilisant le schéma magnétique équivalent de la figure 7 et en tenant compte de la non linéarité des différentes réluctances. Ces dernières sont calculées à partir de la formule classique :

$$
R=\frac{1}{\mu_{0} \mu_{r}} \frac{l}{S}
$$

avec $l$ la longueur du circuit magnétique considéré et $S$ la section de passage de flux.

L'organigramme général de calcul des performances globales de l'actionneur est donné à la figure 22 .

\section{Validation expérimentale}

4.1. Description Du BANC D'ESSAI. - Le prototype linéaire testé destiné à la domotique est de petites dimensions (voir Tab. II). Les efforts statiques développés sont faibles. Pour des mesures fiables et précises (problèmes de frottements) nous avons effectué les mesures d'effort par réaction sur un bâti suspendu par des fils (Fig. 23). Avec cette méthode nous avons obtenu des résultats relativement précis et répétitifs.

La fiabilité et les caractéristiques sont satisfaisantes. Un "étalonnage" a permis de définir ses incertitudes de mesure En effet, ce banc permet d'effectuer des mesures d'effort jusqu'à $100 \mathrm{~N}$, 


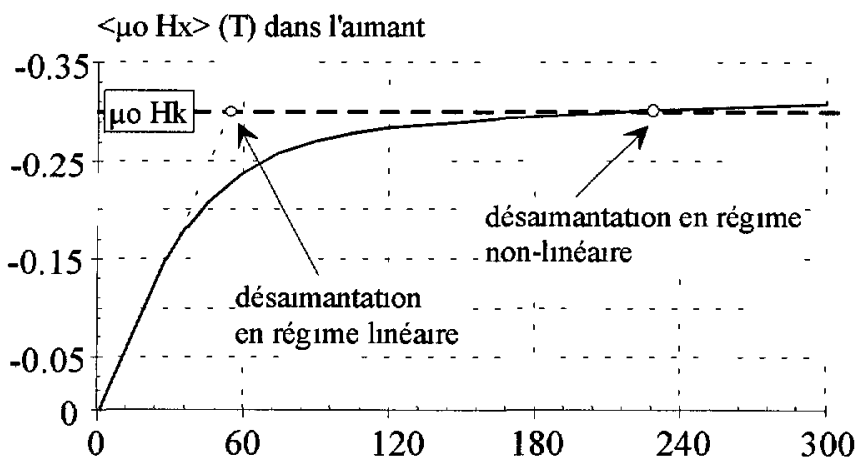

a)

Force magnétomotrice applıquée $(\mathrm{kAt} / \mathrm{m})$<smiles>[3H]</smiles>
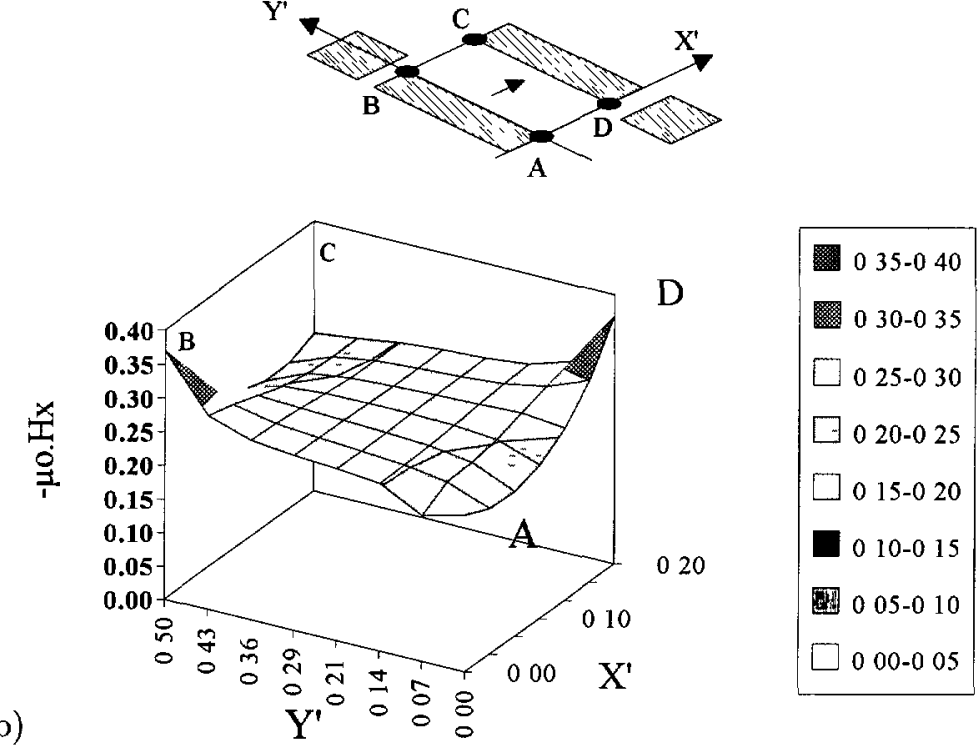

Fig. 21. - a) Variation de la moyenne volumique du champ démagnétisant suivant l'axe $X^{\prime}$ dans l'aimant en fonction de la force magnétomotrice $\left(\mu_{0} H_{k}=0,30 \mathrm{~T}, \lambda=0,5, a=0,5, s=0,3, t=0,4\right.$, $e=0,04)$ b) Représentation du champ démagnétisant suivant l'axe $X^{\prime}$ dans l'aimant $(n I=32 \mathrm{kA} / \mathrm{m}$, $\left.\mu_{0} H_{k}=0,30 \mathrm{~T}, \lambda=0,5, a=0,5, s=0,3, t=0,4, e=0,08\right)$.

[Variation of average value of magnetic induction distribution in magnet's $X^{\prime}$ axis as function of ampere-turns for $\mu_{0} H_{k}=0.30 \mathrm{~T}, \lambda=0.5, a=0.5, s=0.3, t=0.4, e=0.04$. b) Magnetic induction distribution in magnet's $X^{\prime}$ axis for $n I=32 \mathrm{kA} / \mathrm{m}, \mu_{0} H_{k}=0.30 \mathrm{~T}, \lambda=0.5, a=0.5, s=0.3, t=0.4$, $e=0.08$.]

et possède une course utile supérieure à $50 \mathrm{~mm}$. La précision de mesure de l'effort de l'ensemble est satisfaisante $(0,1 \%$ de la pleine échelle) et permet la mesure de faibles efforts de détente inférieurs au Newton. Il est d'autre part entièrement automatisé (mesures et déplacements). Il permet ainsi d'obtenir des formes d'effort en régime statique ou en dynamique soit sur une table traçante, soit sous forme de fichier informatique.

Remarque : Dans l'application nous concernant, la vitesse de déplacement correspondante étant inférieure à $0,2 \mathrm{~m} \mathrm{~s}^{-1}$, le circuit magnétique est massif. Il est néanmoins possible, pour des 


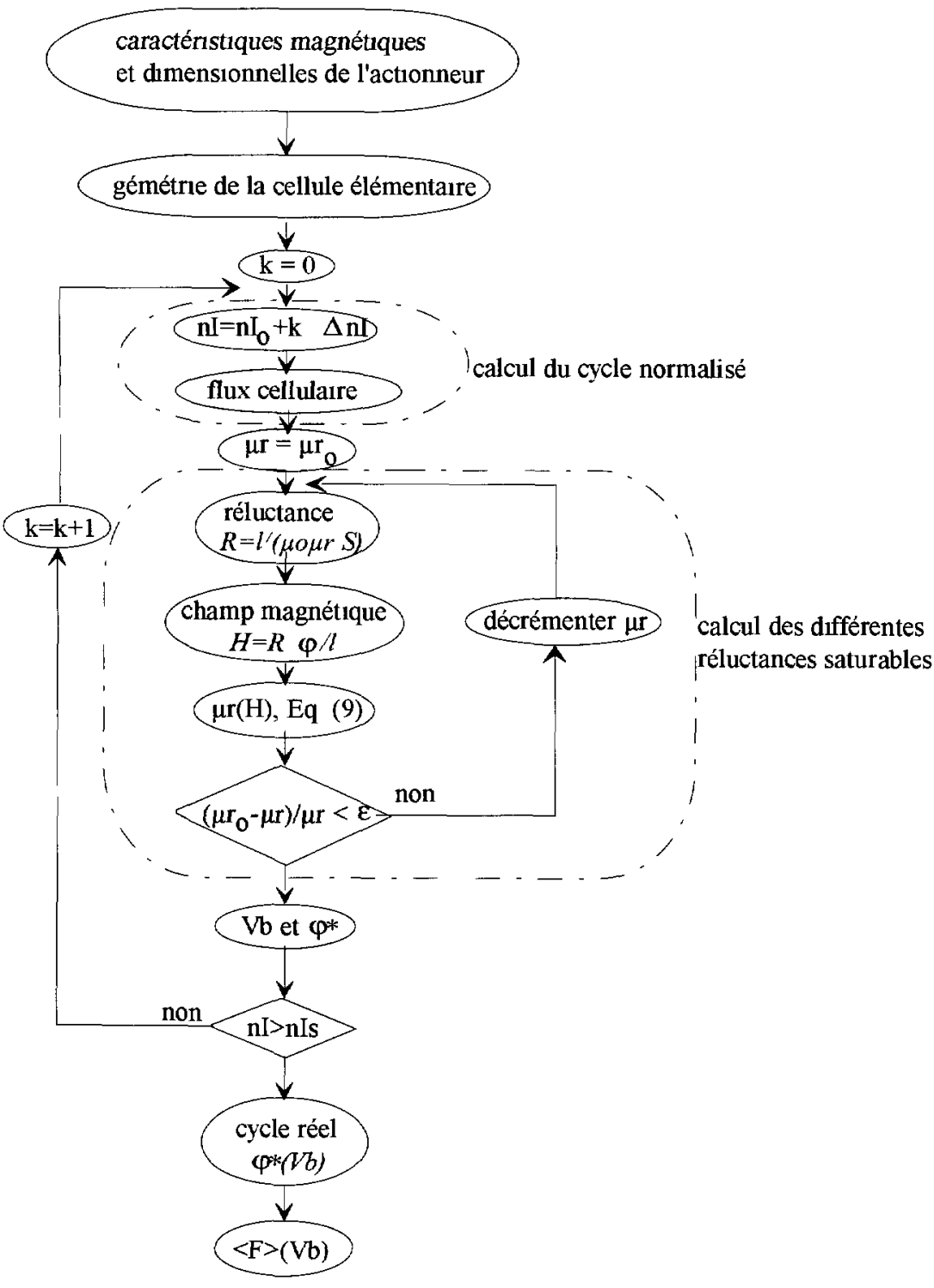

Fig 22. - Organigramme de calcul des performances globales de l'actionneur.

[Global performances organıgram.]

applications à haute vitesse de déplacement, de réduire l'influence des courants de Foucault par l'utilisation de circuit magnétique en poudre de fer.

4.2. RÉSUltats EXPÉRIMENTAUX. - Nous pouvons maintenant comparer les résultats de calculs avec des mesures directes effectuées sur un prototype. Dans ce cas, il faut tenir compte des circuits de retour ainsi que des fuites magnétiques extérieures aux cellules, pré-calculées par éléments finıs (Fig. 8). Les résultats obtenus sur une phase d'un actionneur dont les dimensions sont données dans le tableau II, montrent une assez bonne corrélation avec les mesures 
Tableau II. - Caractéristıques du prototype testé.

[Tested prototype characteristics.]

\begin{tabular}{|l|c|}
\hline Caractéristiques & Valeurs réelles (valeurs réduites) \\
\hline Aimants permanents (ferrites à $\left.80^{\circ} \mathrm{C}\right)$ & $B_{\mathrm{r}}=0,35 \mathrm{~T}, \mu_{0} H_{k}=0,30 \mathrm{~T}$ \\
Largeur aimants & $6 \mathrm{~mm}(t=0,3)$ \\
Hauteur aimants & $35 \mathrm{~mm}(a=0,7)$ \\
Pas dentaire réel & $20 \mathrm{~mm}(\lambda=0,4)$ \\
Largeur dents & $6 \mathrm{~mm}(s=0,3)$ \\
Nombre de cellule élémentaires & 3 \\
Hauteur de la cellule & $50 \mathrm{~mm}$ \\
Profondeur de cellule & $10 \mathrm{~mm}$ \\
Jeu mécanique & $0,5 \mathrm{~mm}(e=0,01)$ \\
\hline
\end{tabular}

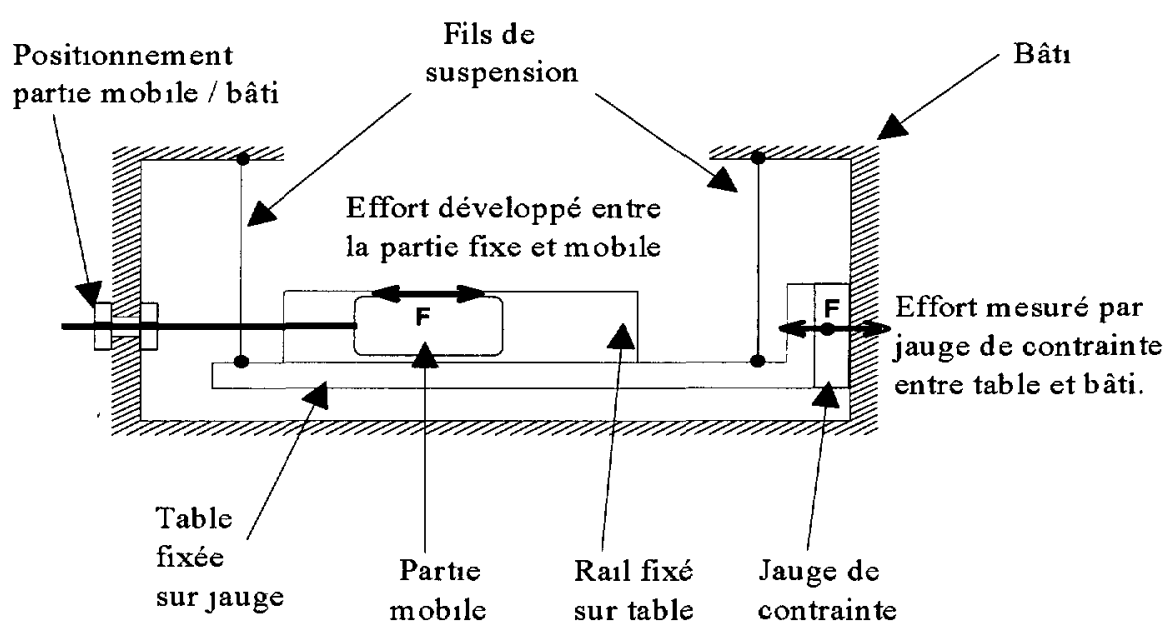

Fig. 23. - Banc d'essal et principe de la mesure par réaction.

[Testing bench and effort measure principle.]

(erreur $<5 \%$ en régime linéaire et $10 \%$ en non linéaire) tant sur le cycle flux/ampères-tours que sur les efforts moyens (Figs. 24 et 25).

Remarque : L'effort développé par l'actionneur testé est relativement faible comparativement aux valeurs optimales obtenues précédemment (Fig. 20). Ceci est dû à la faible valeur des ampères-tours imposés dans le cas des essais. En effet, la force magnétomotrice normalisée imposée est de l'ordre de $16 \mathrm{kA} \mathrm{tm}^{-1}$ (pour $V_{\mathrm{b}}=800 \mathrm{At}$ ). Nous sommes par conséquent loin des possibilités de l'actionneur en termes de limite de désaimantation.

\section{Conclusion}

Cet article a tout d'abord présenté un actionneur à commutation de flux et son principe de fonctionnement. Une modélisation de structures de machines à bobinage global ou multicellulaire en séparant différents éléments caractéristiques a été proposée. Cette modélisation allie 


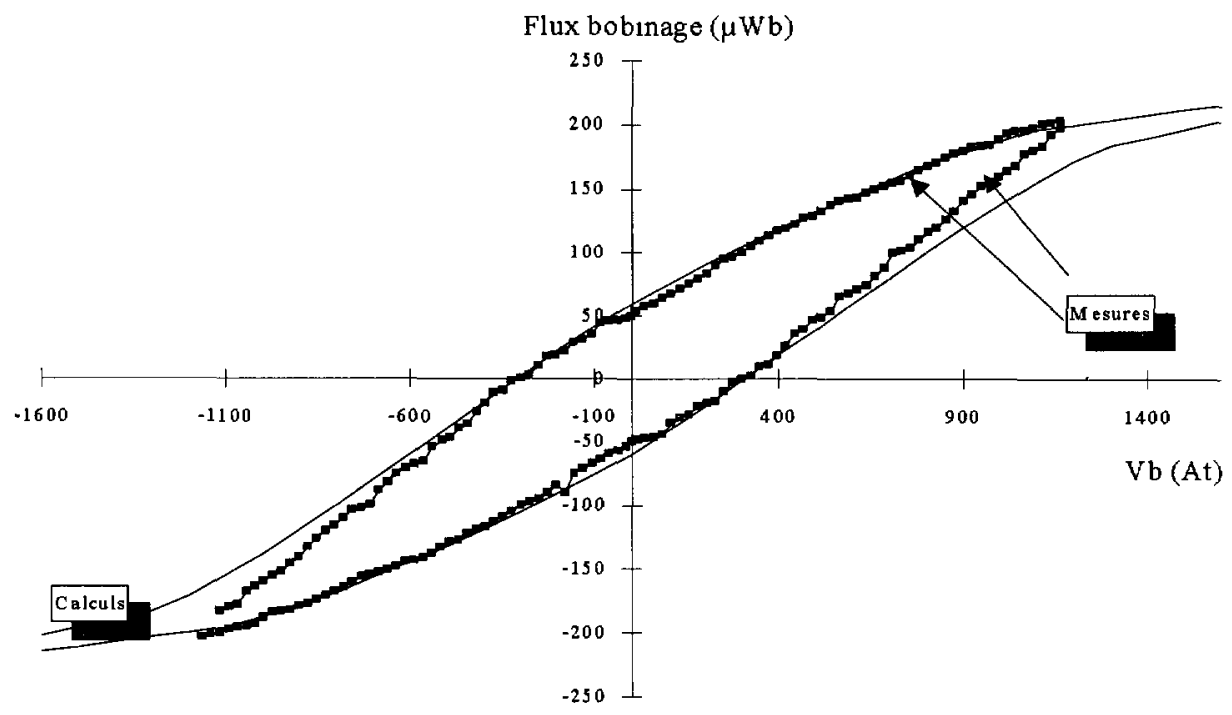

Fig. 24. - Caractéristiques flux/ampères-tours, calculées et mesurées pour une phase en conjonction. [Comparison of the experimental and theoretical results of the magnetic characteristics for conjunction position.]

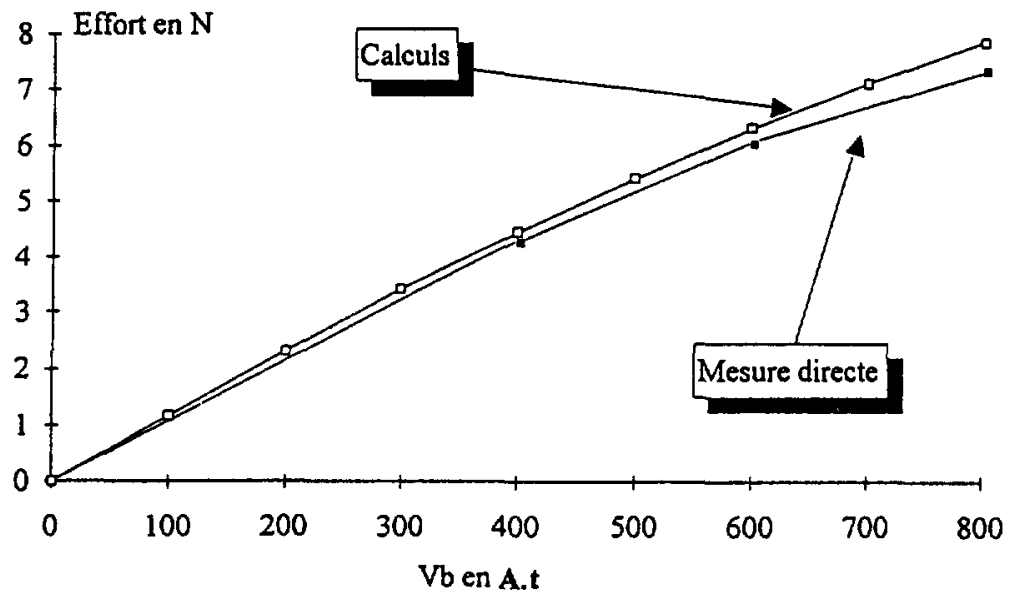

Fig. 25. - Efforts développés en fonctıon de la force magnétomotrice, mesurés et calculés.

[Comparison of the experimental and theoretıcal results of the average effort as function of $\mathrm{m} \mathrm{m}$.]

le calcul de champ par éléments finis dans la zone active et le calcul analytique à partir de réluctances saturables dans la zone de retour de flux.

La principale caractéristique étant la pression tangentielle moyenne développée, celle-ci a été calculée à partir de l'énergie convertie sur un pas dentaire. La détermination du cycle de fonctionnement, présentant des fortes non-linéarités, est effectuée par une méthode à pas adaptatif minimisant ainsı le nombre de points de calcul tout en gardant une précision satisfaisante. 
Il est à noter que la méthode présentée reste applicable pour toutes les structures électromagnétiques saturables tournantes et linéaire telles que les machines à réluctance variable.

À partir de cette méthode, une étude paramétriques intrinsèque a été ensuite effectuée. Les résultats ont permis de déterminer, en fonction du courant imposé, la géométrie optimale maximisant la pression moyenne. Ainsi, les courbes présentées ont montré qu'il était possible d'obtenir une pression tangentielle moyenne importante de l'ordre de $5 \mathrm{~N} \mathrm{~cm}^{-2}$ et ce avec des aimants économiques du type ferrites (pour un fonctionnement en limite de désaimantation).

La confrontation des résultats théoriques aux mesures effectuées sur un prototype d'actionneur linéaire développé au LESIR a montré une très bonne corrélation compte tenu des non-linéarités et des effets tridimensionnels non négligeables.

Il reste cependant à effectuer une analyse paramétrique avec d'autres types d'aimants (Nd$\mathrm{FeB}$, alliés ou non) permettant d'analyser leur influence notamment sur le rapport de concentration de flux. D'autre part, la prise en compte de l'échauffement en cycles, de la forme réelle du courant d'alimentations, des courants de Foucault et enfin de la désaimantation directement dans le processus d'optimisation (couplage fort) permettra une conception globale actionneurconvertisseur.

\section{Annexe}

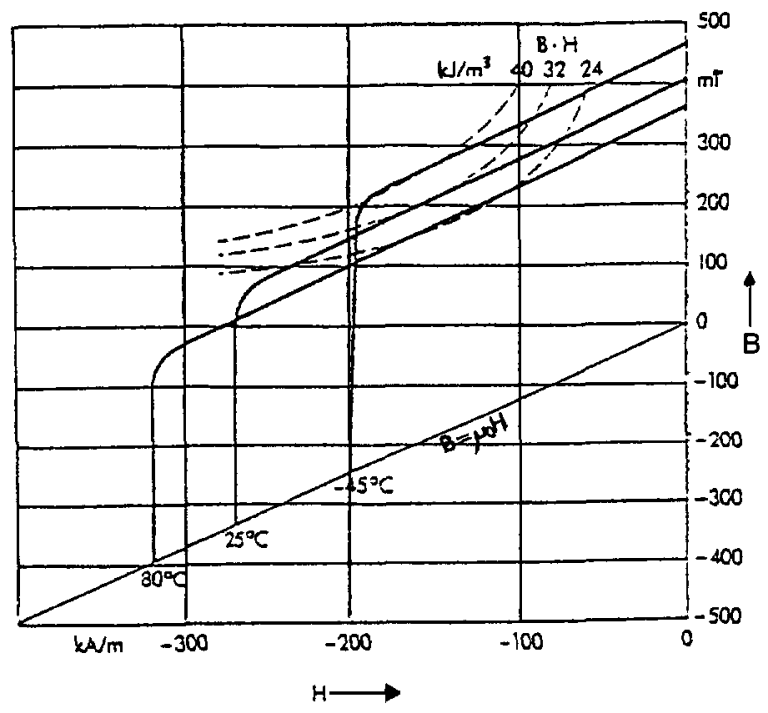

Fig 26. - Caractérıstiques magnétiques des aimants utilisés (Koerox 420 de chez Wıdıa).

[Magnetic characteristic of magnet used.] 


\section{Bibliographie}

[1] Multon B., De nouvelles possibilités avec les moteurs à alimentation électronique dans les applications de grande diffusion, $R G E$ (janvier 1994) 11-19.

[2] Lajoie-Mazenc M., Structures, alimentations et commandes des machines à aimants, Journées 3EI' 95 : Les moteurs synchrones et leurs applications industrielles (Paris 30-31 mars 1995).

[3] Cathey J.J. and Richter E., A high force density linear switched reluctance machine, Proc. IEEE I (1993) 251-257.

[4] Rauch S.E., Magnetic circuit properties of the flux switch inductor alternator, AIEE Trans. 77 II (November 1958) 307-312.

[5] Tia P., Matt D., Poulot P. and Regord C., Predetermination of permanent magnet reluctant machine with flux concentration, Proc. IMACS 1/3 (1996) 1131-1136.

[6] Prevond L., Étude, expérimentation et modélisation de structures hybrides innovantes tournantes et linéaires, Thèse de Doctorat (Conservatoire national des arts et métiers Paris, décembre 1994).

[7] Lucidarme J., Multon B. et Prevond L, Actionneurs hybrides monophasés à commutation de flux, Brevet Français n ${ }^{\circ}$ F94/12063 (10/10/94). Demande de Brevet Europeen $\mathrm{n}^{\circ} 93402266.1$ (octobre 1995).

[8] Prevond L., Lucidarme J. and Multon B., Low cost flux switching linear hybrid actuator, Proc. ICEM 94 II (septembre 1994) 317-322.

[9] Ben Ahmed A., Contribution à la conception des machines synchrones à aimants permanents et à bobinage global, Thèse de Doctorat Paris VI (janvier 1994).

[10] Tormey D., Torrey D. and Levin P.L., Minimum Airgap-Permeance Data for DoublySlotted Pole Structures Common in Variable-Reluctance-Motors, Proc. IEEE Seattle (1990) 196-200.

[11] Bastos J.P., Calcul de champs magnétiques par une méthode d'éléments finis à 2 et 3 dimensions - contribution à la détermination des caractéristiques de machines à réluctance variable polydiscoïdes, Thèse de doctorat d'état, université de Paris VI (mars 1984).

[12] Ben Ahmed A., Lucidarme J. et Desesquelles P.F., Méthode semi-numérique de prédimensionnement des machines à aimants permanents et à bobinage global, J. Phys. III France 5 (1995) 703-725. 